\title{
Weighted Gene Coexpression Network Analysis Reveals Cancer Stem Cell-Associated Metabolic Gene Signature in Glioma
}

\section{Yan Tang ( 327194072@qq.com )}

Southwest Medical University https://orcid.org/0000-0002-5513-6453

\section{Yao Jiang}

Southwest Medical University

Dan Zhang

Southwest Medical University

Jia Fan

Southwest Medical University

Juan Yao

Southwest Medical University

Zhihua Zuo

Southwest Medical University

\section{Yiqin Li}

Southwest Medical University

\section{Hualin Tao}

Southwest Medical University

\section{Yongcan Guo}

Southwest Medical University

\section{Research Article}

Keywords: Glioma, IDH mutation, Cancer cell stemness, WGCNA, Prognosis

Posted Date: February 18th, 2021

DOl: https://doi.org/10.21203/rs.3.rs-205111/v1

License: (c) (1) This work is licensed under a Creative Commons Attribution 4.0 International License. Read Full License 


\section{Abstract}

Background: Isocitrate dehydrogenase (IDH) mutant glioma patients have a favorable prognosis, accompanying with metabolic alterations and glioma cell dedifferentiation. Recently, mRNA expressionbased stemness index (mRNAsi) characteristic relation to IDH status of gliomas has yet illuminated. Thus, we aimed to establish a cancer stem cell-associated metabolic gene signature for risk stratification of gliomas.

Methods: The glioma samples came from The Cancer Genome Atlas (TCGA) and the Chinese Glioma Genome Atlas (CGGA) databases. Next, we performed the differential expression analysis between IDH mutant and $I D H$ wild-type gliomas and also conducted weighted gene correlation network analysis (WGCNA) for determining the modules associated with cancer stem cell trait. Subsequently, multivariate Cox regression analysis with the Akaike information criterion (AIC) algorithm was employed to establish a stemness-related metabolic gene signature, which was validated using time-dependent receiver operating characteristic (ROC) curves and concordance index (C-index). Also, we developed a nomogram based on clinical traits and prognostic model. Additionally, according to the results of immunohistochemistry (IHC) staining, the protein levels of gene signature were consistent with the genes expression's direction.

Results: Low expression of mRNAsi was capable of predicting the unfavourable OS of gliomas with a 5year survival rate of $14.08 \%$. The blue module and its 1466 genes were pertinent to mRNAsi characteristic. Next, Kaplan-Meier (KM) survival curves revealed that cancer stem cell-associated metabolic genes exerted impact on gliomas' prognosis. Subsequently, univariate and multivariate Cox regression analyses were implemented, and gene signature (LCAT, UST, GALNT13, and SMPD3) was constructed, with C-index of 0.798 (95\%Cl: 0.769-0.827). Notably, the prognostic model presented a superior predictive value for gliomas' survival, with the area under the curve (AUC) of ROC curves at 1year, 3-year as well as 5-year time-point of $0.845,0.85$ and 0.811 , respectively. And forest plot uncovered its role as a potential independent predictor for gliomas ( $\mathrm{HR}=2.840,95 \% \mathrm{Cl}: 1.961-4.113, P<0.001)$. Nomogram also presented superior predictive performance for gliomas' OS.

Conclusion: The gene signature ( $L C A T$, UST, GALNT13, and SMPD3) can be used for risk stratification and also can serve as an independent prognostic factor of glioma patients.

\section{Introduction}

Glioma originates from glial cells or precursor cells, and is one of the most common types of primary brain tumors $(1,2)$, with an average annual age-adjusted incidence rate from 2010 to 2014 of 6 per 100,000 in the United States (3). According to the histopathological criteria established by the World Health Organization (WHO), glioma is classified into grade I to grade IV (4). The standard therapy for glioma patients includes maximal surgical resection (2) and radiotherapy and/or chemotherapy as early as possible (5). Although these treatments have been adopted, low grade glioma (LGG) may evolve into higher grade glioma or recur (6), with poor overall survival (OS). More recently, with the popularization of 
microarray chips and high-throughput sequencing, tumor stratification has transformed from morphology to molecular traits (7). At present, due to the presence of the limitations in the early diagnosis of gliomas and prediction of its prognosis, specifically for high-grade glioma (HGG), it is imperative to illuminate the molecular mechanisms of gliomas. In 2006, the WHO have established some molecular biomarkers for sub-classification, including the co-deletion of chromosomal arms $1 p$ and $19 q$, isocitrate dehydrogenase (IDH) mutations, methylated status of the 0-(6)-methylguanine-DNA methyl transferase (MGMT) promoter, alpha thalassemia/mental retardation syndrome X-linked (ATRX) mutations or loss, and telomerase reverse transcriptase (TERT) promoter mutations (6). IDH mutations appear to confer favorable OS in glioma patients, regardless of WHO grades $(8,9)$. It may be of interest to note that IDHI $(\mathrm{R} 132 \mathrm{H})$ mutation alters the metabolic flux of isocitrate and a-ketoglutarate ( $\mathrm{a}-\mathrm{KG})$, and induces widespread metabolic reprogramming, thought to be common characteristics of tumorigenesis and serve roles in gene expression, cell differentiation and the tumor microenvironment (10), and provokes some compensatory metabolic changes for maintaining biosynthetic requirements during glioma cells proliferation (11-13). The metabolic alterations are considered to propel tumorigenesis and block differentiation by influencing epigenetics (14-16). Additionally, a population of undifferentiated cells with stemness in glioma has been determined as a main factor affecting tumor initiation, development, recurrence and invasion (17). In essence, the development of cancer involves in cells gradual loss of the differentiated phenotype and acquisition of progenitor cell-like, or stem cell-like characteristics, which more readily cause the tumor cell migration to distant organs, resulting in unfavorable prognosis and chemotherapy-resistance (18-20). Recently, it has been reported that the characteristics of tumor stem cells were investigated by bioinformatics methods. Malta et al. employed a novel one-class logistic regression machine-learning algorithm (21) to identify transriptome and epigenetic traits derived from normal pluripotent stem cells and their differentiated progeny in The Cancer Genome Atlas (TCGA) database (22). They reported two stemness indices, including a gene expression-based stemness index (mRNAsi), representative of the degree of dedifferentiation of tumors (23), and an epigenetically regulated-mRNAsi (EREG-mRNAsi) (24). The scope of these two indices ranges from 0 to 1 , where the closer the value to 1 , the stronger the stem cell characteristics of tumors are. In the previous study, mRNAsi and EREG-mRNAsi values were demonstrated to be negatively correlated with gliomas' pathology and clinical traits. They ascribed the phenomenon in high frequency of $I D H$ mutations in glioma patients. Since IDH mutations reduce cell differentiation, mRNAsi values were higher in the IDH mutant subset. However, the cancer stem cell-related metabolic genes associated with IDH mutations in gliomas have yet specifically elucidated. Thus, we aimed to establish a prognostic model pertinent to metabolic reprogramming and dedifferentiated status in $I D H$ mutant glioma, for risk stratification in gliomas.

In the present study, we have determined that glioma patients with higher mRNAsi score had poor prognosis via Kaplan-Meier (KM) method and log-rank test. Next, we performed differential expression analysis between IDHmutant and IDH wild-type gliomas after merging the TCGA-GBM and TCGA-LGG cohorts and removing their batch effects. Subsequently, weighted gene correlation network analysis (WGCNA) was employed to identify the modules closely associated with the mRNAsi trait. Then, we 
selected genes related to metabolism and cancer stem cell characteristic as core genes. Next, univariate Cox regression analysis revealed their relationship to gliomas' prognosis, where the genes with $P$-value less than 0.05 were used to perform multivariate Cox regression analysis with Akaike information criterion (AIC) algorithm for constructing a prognostic model (including LCAT, UST, GALNT13 and SMPD3 genes). In addition, we developed a nomogram for clinicians and validated its predictive performance using calibration curves based on bootstrap method. Our findings may provide deep insight into risk stratification of gliomas, illuminate the potential molecular traits of $I D H$ mutant gliomas from metabolic reprogramming and cancer stem cell aspects, and be conducive to target therapies for glioma patients in the near future.

\section{Materials And Methods}

\section{Data Sources and Processing}

The RNA-sequencing data (FPKM) of glioblastoma (GBM) $(n=169)$ and LGG $(n=529)$ as well as its clinical data were downloaded from the TCGA database (https://portal.gdc.cancer.gov). Subsequently, the Ensembl database was employed to integrate the EnsembI ID into the official gene symbols, and expression data was log2 converted. The values of mRNAsi and EREG-mRNAsi of all glioma patients, including LGG and GBM, were obtained from https://www.ncbi.nlm.nih.gov/pmc/articles/PMC5902191/ (24). Furthermore, the clinical information (gender, age, status, overall survival, IDH status, tumor grade, histological subtypes, MGMT status and other mutation information) of TCGA-GBM and TCGA-LGG patients was downloaded from the cBioPortal for Cancer Genomics (www.cbioportal.org). Additionally, external cohorts of mRNAseq_693 $(25,26)$ and mRNAseq_325 $(27,28)$ from the CGGA database (www.cgga.org.cn/) were acted as external validation datasets, where only primary glioma samples were selected. The combat function of the "sva" package was employed to remove batch-batch differences (29).

\section{Differential Expression Analysis}

After eliminating batch effects, the RNA-seq data of TCGA-GBM and TCGA-LGG were integrated into a single glioma group. According to IDH mutation status, we used "limma" package to implement differential expressed analysis between IDH wild-type and IDH mutant gliomas. The differential expressed genes (DEGs) should met the following criteria: $\mid \log _{2}$ Fold-Change $\left(\log _{2} F C\right) \mid>1$ and adjusted $P<0.05$.

\section{The Relationship Between mRNAsi and Clinical Traits}

An unpaired t-test was applied to compare differences in the mRNAsi values of glioma samples. One-way analysis of variance (ANOVA) used to compare comparison with significant differences in the mRNAsi among variable groups. X-tile software (Version 3.6.1) (30) was applied to determine the optimal cut-off values in survival data, then we classify glioma patients into high and low expression groups. This software undertook enumeration method, meaning it groups different values as cut-off for statistical analysis, and adopted the result with the smallest $P$-value as the optimal truncation value. Based on the 
best cut-off values, KM survival curves of two groups were depicted using the "survminer" package, and the $P$-value was calculated using the log rank test.

\section{Weighted Gene Coexpression Network Analysis}

Establishment of a Coexpression network

Construction of significant modules was completed using the "WGCNA" package, which was used to find the correlations between genes (31). We selected a total of 2441 DEGs to establish a coexpression network. The formula of the constructed adjacency matrix was as follows: $a_{i j}=|\operatorname{cor}(i, j)|^{\beta}(22)$, where cor $(\mathrm{i}, \mathrm{j})$ represents the Pearson correlation coefficients between gene $\mathrm{i}$ and gene $\mathrm{j}, \mathrm{a}_{\mathrm{ij}}$ represents the adjacency between gene $i$ and gene $j$ and $\beta$ is the soft threshold. The "pickSoftThreshold" function of the "WGCNA" package was employed to determine the value of $\beta$, making the network close to the scale-free network distribution on the basis of connectivity (31). Subsequently, an adjacency matrix and topological overlap matrix (TOM) was constructed according to the $\beta$ value.

Identification of significant modules

Next, the dynamic shear method was applied to classify consistently expressed genes into the same modules (32), with a minimum of 50 genes in a module. To improve the merging of modules with high similarity, we selected 0.25 as a cut-off value (33). Additionally, we aimed to identify the modules with the highest relation to sample features. Then, the correlation coefficients and $P$ value between modules and clinical traits (mRNAsi and EREG-mRNAsi values) were calculated, and the top ranking modules were selected as the most essential modules. Subsequently, we calculated the gene significance (GS) and module member (MM) (22). The higher the GS value of genes, the stronger their association with the stem index.

Functional enrichment analysis of the modules

We extracted the genes in the most significant modules and performed functional enrichment analysis using the clusterProfiler package (34), including gene ontology (GO) terms and Kyoto Encyclopedia of Genes and Genomes (KEGG) pathways enrichment analyses. An adjusted $P<0.05$ acted as a cut-off value.

\section{Metabolism-related Genes}

The seventy metabolism-related gene sets derived from the KEGG pathway database and a total of 1466 metabolic genes were obtained from https://figshare.com/s/d2a968666f6f4c9ea224 (35). To investigate the genes closely related to metabolic reprogramming and cancer stem cell trait, we screened out the intersected region in the most significant modules and KEGG metabolic pathway gene lists, which were regarded as core genes, and Venn plot showed these genes.

\section{Survival and univariate Cox analyses}


Adopting optimal truncation values calculated by X-tile software, we divided glioma into high and low core genes expression groups. Next, KM survival curves revealed that their expression levels' exerted impact on gliomas' OS. And univariate Cox regression analysis was used to identify core genes significantly influencing glioma patients' prognosis, where genes with $P<0.05$ were selected for further analysis.

\section{Establishment of a Prognostic Signature}

Multivariate Cox regression analysis based on AIC algorithm (36) was used to construct a prognostic model. Additionally, the risk score formula was composed of the product of expression level of each core gene and their coefficients derived from multivariate Cox regression analysis. As shown in Eq. 1, Gi is the expression level of gene $i$, coefficient $t_{i}$ is the coefficient of gene $i$ and $n$ is the number of core genes in the prognostic model. Given that 5-year survival rate is of significance for patients suffering from tumors, we utilized optimal cut-off value of risk score on time-dependent receiver operating characteristic (ROC) curve of evaluating 5-year survival to classify patients into high and low risk groups (37). Subsequently, we used KM curves to show the relationship between the risk score and OS of gliomas.

Risk score $=\sum_{i=1}^{n} G_{i} \times$ coefficient $_{i}$

\section{Evaluation of the Prognostic Value of a Four-gene-based Signature}

Next, time-dependent ROC curves (38) (1-year, 3-year and 5-year) were employed to evaluate the predictive capability of the risk score model for glioma patients' prognosis. Also, the concordance index (C-index) was used to assess the predictive accuracy of this prognostic model, namely the discrepancy between the true value and predicted value of model (39). The C-index ranges from 0.5 to 1.0 , where $0.50-0.70$ refers to low accuracy, $0.71-0.90$ represents medium accuracy, and higher than 0.90 is representative of high accuracy. Risk score, clinical characteristics and molecular factors, including gender, age, glioma grade, $I D H$ mutation, methylation of $M G M T$ promoter and histological subtypes were analyzed using multivariate Cox regression analysis. The factors with $P<0.05$ were considered to be independent prognostic indicators. Subsequently, a nomogram of 1-year, 3-year and 5-year OS was developed using the "rms" package (40) in the RStudio platform (41). Similarly, calibration curves was used to verify the accuracy of the nomogram in predicting glioma survival via the bootstrap resampling method (42).

\section{External Validation of Prognostic Value}

We merged the expression data of mRNAseq_693 and mRNAseq_325 cohorts and removed the batch effects, then verified the prognostic value of this prognostic model. The risk score was calculated based on Eq. 1, where glioma patients were divided into high and low risk groups according to optimal cut-off values on the ROC curve at 5-year time-point. Additionally, we also constructed a nomogram and perform calibration analysis for validation of its predictive value, beneficial for clinicians to make decisions. 


\section{Immunohistochemistry}

The human protein atlas database (HPA, https://www.proteinatlas.org/) (43) was employed to detect the protein expression of core genes, where the researchers used highly specific antibodies and adopted immunohistochemistry $(\mathrm{IHC})$ method to compare the protein levels between normal and tumor tissues. We chose the cerebral cortex tissues, LGG and HGG samples to visualize their expression from the protein aspect.

\section{Statistical Analysis}

All analyses were implemented in RStudio software (Version 3.5.0). Firstly, selection of DEGs was performed via the Wilcoxon test (44). Second, X-tile software was used for means testing using a standard ANOVA calculation on two-population (high vs. low groups) cut-points (30). Thirdly, Pearson's chi-square tests were employed to investigate the correlation among core genes. Additionally, KM curves were generated by the "survminer" package and $P$-values were calculated via the Wilcoxon log rank test. Two-tailed $P$-values less than 0.05 were considered statistically different.

\section{Results}

\section{Clinical Characteristics of Datasets}

A total of 624 glioma samples from the TCGA database with sufficient IDH status were included for differential expression analysis. However, 616 samples were used for construction of the prognostic model due to eight patients without sufficient survival data. Meanwhile, there were 588 primary glioma samples from the mRNAseq_693 and mRNAseq_325 cohorts as an external validation cohort. And Table 1 showed the clinical characteristics of glioma patients in two databases. The flow diagram of the present study was provided in Fig. 1. 
Table 1

Clinic pathological characteristics of glioma samples from the TCGA and CGGA databases

\begin{tabular}{|c|c|c|}
\hline \multirow[t]{2}{*}{ Characteristics } & Training cohort & Validation col \\
\hline & $(T C G A, N=616)$ & (CGGA, $N=5$ \\
\hline \multicolumn{3}{|l|}{ Age (\%) } \\
\hline$\leq 46$ & $321(52.11)$ & $353(60.03)$ \\
\hline$>46$ & $295(47.89)$ & $234(39.80)$ \\
\hline NA & - & $1(0.17)$ \\
\hline \multicolumn{3}{|l|}{ Gender (\%) } \\
\hline Female & $267(43.34)$ & $241(40.99)$ \\
\hline Male & $349(56.66)$ & $347(59.01)$ \\
\hline \multicolumn{3}{|l|}{ Grade (\%) } \\
\hline G2 & $239(38.80)$ & $208(35.37)$ \\
\hline G3 & $256(41.56)$ & $169(28.75)$ \\
\hline G4 & $121(19.64)$ & $211(35.88)$ \\
\hline \multicolumn{3}{|l|}{ IDH_status (\%) } \\
\hline IDH_mut & $411(66.72)$ & $309(52.55)$ \\
\hline IDH_wt & $205(33.28)$ & $279(47.45)$ \\
\hline \multicolumn{3}{|c|}{ MGMT_status (\%) } \\
\hline Methylated & $459(74.51)$ & $187(31.80)$ \\
\hline Unmethylated & $149(24.19)$ & $332(56.46)$ \\
\hline NA & $8(1.3)$ & 69 (11.74) \\
\hline
\end{tabular}

TCGA, The Cancer Genome Atlas; CGGA, Chinese Glioma Genome Atlas; G2, grade 2; G3, grade 3; G4, grade 4; IDH, Isocitrate dehydrogenase; IDH_mut, IDH mutation; IDH_wt, IDH wild type; NA, not available.

\section{Correlation Between mRNAsi and Clinical Traits in Glioma Patients}

The relationships between mRNAsi and some clinical information were investigated. As shown in Fig. 2C, mRNAsi was higher in glioma patients with IDH mutations $(P<0.0001)$. Additionally, patients older than 60 years old were associated with low levels of miRNAsi (Fig. $2 A, P<0.0001$ ), while there was no obvious difference in gender (Fig. 2B, $\mathrm{P}=0.69$ ). Intriguingly, mRNAsi levels gradually decreased as the glioma 
grade increased (Fig. 2D, $\mathrm{P}<0.0001)$. In addition, we observed that glioma patients with low mRNAsi score had an unfavorable prognosis (Fig. $2 \mathrm{E}, \mathrm{P}<0.0001$ ), with an optimal cut-off value of 0.30 calculated by X-tile software. However, the difference in EREG-mRNAsi was no statistically significant (Fig. 2F, $\mathrm{P}=$ $0.13)$. Hence, we selected mRNAsi as the main trait for the next analysis.

\section{Identification of Differentially Expressed Genes (DEGs)}

Considering that mRNAsi was closely associated with glioma OS and the difference in IDH wild-type and IDH mutant glioma was significant, our aim was to investigate the DEGs between the two groups. Subsequently, the "limma" package was adopted to conduct differential expression analysis in 207 cases with wild-type IDH and 417 cases of $I D H$ mutant gliomas after removing batch-batch differences. Using the absolute value of $\log _{2}$ FoldChange $\left(\mid \log _{2} \mathrm{FCl}\right)>1.0$ and an adjusted $P$-value $(\operatorname{adj} . P)<0.05$ as the cut-off value, we identified 2441 DEGs (Supplementary Table 1), with up-regulation of 1616 and down-regulation of 825 DEGs.

\section{WGCNA}

Essential modules

After eliminating four outlier samples (Supplementary Figure 1), a total of 2441 DEGs were used to establish a coexpression network using the "WGCNA" package. The value of $\beta$ was six based on the scale-free topology distribution, making the $\mathrm{R}^{\wedge} 2$ reach 0.88 (Figure $3 \mathrm{~A}$ ). We selected 0.25 as the MEDissThres value, resulting in eight modules (black, blue, brown, green, grey, pink, red and yellow), with the gene number contributing to each ranging from 67 to 1132 (Figure 3B). Each color module represents a common gene expression module. Next, in a correlation analysis of these modules and glioma traits (mRNAsi and EREG-mRNAsi), we found that the blue $\left(R^{\wedge} 2=0.70, P<0.0001\right.$, Figure $3 C$ ) module was obviously positively correlated with mRNAsi and the brown ( $R^{\wedge} 2=-0.6, P<0.0001$, Figure $3 C$ ) module was negatively correlated with mRNAsi. Then, by application of intramodular analysis, we determined that the blue module was characterized by higher GS and MM as the most significant module (cor $=0.83, P=1.3 \mathrm{e}-$ 96, Figure 3D). Ultimately, the Venn diagram showed that a total of seven genes, including LCAT, UST, IPMK, NEU4, GALNT13, PDE6B and SMPD3 genes, were cancer stem cell-pertinent metabolic genes (Figure $3 \mathrm{E}$ ), designated as core genes. In addition, the correlation analysis among these genes revealed that they had medium positive correlations with each other at the mRNA expression level except $P D E 6 B$, which was negatively correlated with other genes (Supplementary Figure 2A). Meanwhile, higher PDE6B expression was observed in $I D H$ wild-type glioma patients via a hierarchical clustering heatmap (Supplementary Figure 2B).

Functional enrichment analyses

To further describe the functions of the genes in the blue module, gene ontology (GO) terms and Kyoto Encyclopedia of Genes and Genomes (KEGG) pathway enrichment analyses were undertaken. As shown in Figure 4, the GO chord plots of GO term and KEGG pathway enrichment analyses indicated that the blue module's genes were mainly enriched in regulation of the membrane potential, postsynapse, ion 
channel activity (GO terms, Figure $4 A-C$ ), neuroactive ligand-receptor interaction and the cAMP signaling pathway (KEGG, Figure 4D).

\section{Survival Analysis}

To explore the prognostic value of core genes in glioma, the best truncation values were determined by Xtile software and glioma patients were classified into high/low expression groups. Of note, KM survival curves denoted that most of the core genes with low expression were associated with unfavorable OS ( $P$ $<0.0001$, Figure 5A-F). By contrast, high expression of $P D E 6 B$ was associated with a worse prognosis for glioma patients $(P<0.0001$, Figure $5 \mathrm{G})$. Next, we included all core genes for univariate Cox regression analysis, where the results demonstrated that six core genes (HR $<1.0, P<0.0001$, Table 2$)$ were protective factors of glioma, other than PDE6B, which had an HR value of $1.257(95 \% \mathrm{Cl}: 1.187-1.33)(P<0.0001$, Table 2), functioning as a risk factor.

\section{Construction of a Prognostic Signature}

Multivariate Cox proportional hazard regression analysis with the AIC algorithm had identified a four gene-based signature, including $L C A T$, UST, GALNT13 and SMPD3 genes (Table 2, Figure 6A), with the coefficients of the four core genes used to calculate the risk score according to equation 1 . The risk score formula was as follows: risk score $=(-0.028787) *$ LCAT expression level $+(-0.024918)$ * UST expression level $+(-0.031937) *$ GALNT13 expression level $+(-0.26647) *$ SMPD3 expression level, which had the minimal AIC value of 2158.69 and C-index of 0.798 (95\%Cl: $0.769-0.827)$. Additionally, survival curve revealed that glioma patients with high risk score had worse OS than low risk patients (Figure 6B, $\mathrm{HR}=5.992,95 \% \mathrm{Cl}: 4.373-8.209, P<0.0001)$. The 5 -year survival rates were separately $8.79 \%$ and $69.2 \%$ in high and low score groups classified by an optimal cut-off value of 1.659003. Furthermore, a GO chord plot demonstrated that four genes were enriched in ammonium ion metabolic process, glycoprotein biosynthetic process, dermatan sulfate biosynthetic process, steroid esterification ( $\mathrm{GO}$ term, category $=$ "BP", Figure 6C) and 0-acyltransferase activity (GO term, category = "MF", Figure 6C).

\section{Assessment of the Prognostic Value of the Four-gene-based Signature}

We used the TCGA cohort to internally verify the prognostic performance of the risk score model. Firstly, the area under the curve (AUC) of the time-dependent ROC curves were $0.845,0.85$ and 0.811 for 1 -year, 3 year and 5-year OS, respectively (Figure 6D). Second, we evaluated whether the four-gene-based signature was an independent prognostic indicator of the glioma. According to the result of univariate Cox regression analysis, we did not take the gender trait (HR=1.113, 95\% Cl: 0.849-1.458, $P=0.439$, Table 3 ) into consideration for performing multivariate Cox regression analysis. Then we used a forest plot to show that the risk score (HR=2.840,95\% Cl: 1.961-4.113, $P<0.001$, Figure $6 \mathrm{E}$, Table 3$)$ was an independent prognostic factor via multivariate Cox regression analysis combining significant clinical traits, such as age ( $\geq 60$ vs. $<60$ ), tumor grade (HGG vs. LGG), MGMT status (unmethylated vs. methylated) as well as histological subtypes (ASTR vs. GBM; ODG vs. GBM; mixed glioma vs. GBM). Intriguingly, compared with GBM, astrocytoma (ASTR, HR=0.401, 95\%Cl: 0.265-0.607, $P<0.001$ ), oligodendroglioma (ODG, HR=0.332, 
95\%Cl: 0.204-0.541, $P<0.001)$ and mixed glioma (HR=0.393, 95\%Cl: 0.233-0.661, $P<0.001)$ were pertinent to better prognosis of glioma patients. Remarkably, age ( $\mathrm{HR}=2.137,95 \% \mathrm{Cl}: 1.511-3.021, P<0.001)$, tumor grade $(\mathrm{HR}=2.461,95 \% \mathrm{Cl}: 1.632-3.713, P<0.001)$ and $M G M T$ status $(\mathrm{HR}=1.680,95 \% \mathrm{Cl}: 1.248-2.261$, $P<0.001)$ were significantly associated with gliomas' prognosis, with a C-index of 0.854 ( $95 \% \mathrm{Cl}$ : 0.830 0.878). Table 3 showed the results from the univariate and multivariate Cox regression analyses. A nomogram integrating the prognostic model and the above mentioned clinical characteristics was developed for predicting the 1-OS, 3-OS, and 5-OS of glioma patients (Figure 6F). Additionally, calibration curves (Figure 6G) were adopted to evaluate the consistency between the predictive values from the nomogram and observed values in 1-OS , 3-OS and 5-OS of glioma patients, showing that the nomogram had superior predictive capacity.

\section{Validation of the Prognostic Model}

We selected a total of 588 glioma patients from the CGGA database as an external validation cohort. Based on equation 1, the risk score of each patient was calculated. Then, KM survival curve revealed that the high risk glioma population had worse OS than low risk patients, grouped by a optimal cut-off value of 0.2926944 , and these results were consistent with the TCGA training set (Figure 7A). And the 5-year survival rates of high and low risk patients were $28.50 \%$ and $66.30 \%$, respectively. The risk distribution, patients survival status as well as the heatmap of the four gene-based signature were also depicted (Figure 7B). Then, the AUC of the ROC curves for 1-year, 3-year and 5-year OS were $0.758,0.763$ and 0.738 , respectively (Figure $7 \mathrm{C}$ ). Also, multivariate Cox proportional hazard regression analysis for the risk score and clinical characteristics (such as age, glioma grade, MGMT status and histological subtypes) was performed, indicating that the risk score ( $\mathrm{HR}=1.423,95 \% \mathrm{Cl}: 1.104-1.834, P=0.0065)$ was an independent prognostic indicator (Figure 7D), with a C-index of being 0.783 (95\% Cl: 0.759-0.807). Additionally, we constructed a nomogram via multivariate Cox regression analysis for the risk score and clinical traits (Figure 7E). We found that ASTR and GBM had higher scores than oligoastrocytoma (OAST) and ODG patients in the nomogram, which might be related to the presence of anaplastic astrocytoma (AASTR) in the ASTR patients. Ultimately, the calibration curves (Figure 7F) discovered that the nomogram had good predictive performance for the 1-OS, 3-OS, and 5-OS of glioma patients.

\section{Comparison of the Four Gene Expression Levels using the CGGA Cohort}

We used the CGGA dataset to compare the expression of the four gene based on IDH status, denoting IDH mutant glioma with higher $\angle C A T$ (Figure 8A, $P<0.0001$ ), UST (Figure 8B, $P<0.0001$ ), GALNT13 (Figure 8C, $P<0.0001$ ) and SMPD3 (Figure 8D, $P<0.0001$ ) levels. Also, we performed survival analyses to determine the impact of the four core genes on glioma survival. The results indicated that these core genes with high expression were involved in favorable prognosis of the glioma (Figure $8 \mathrm{E}-\mathrm{H}, P<0.001$ ).

\section{Immunohistochemistry}

Due to the absence of immunohistochemical results of the LCAT protein in normal brain and glioma tissues in the HPA database, the comparison between the two groups were unavailable. Compared with 
normal cerebellum tissues, the UST, GALNT13 and SMPD3 brown protein granule in LGG and HGG tissues were decreased (Figure 9), indicating that the consistent results of gene and protein expression in three core genes.

TABLE 2 Univariate and Multivariate Cox regression analysis of the core genes

\begin{tabular}{|llllllll|}
\hline \multirow{2}{*}{ Genes } & \multicolumn{2}{l}{ Univariate analysis } & \multicolumn{5}{l|}{ Multivariate analysis } \\
\cline { 2 - 8 } & HR & $95 \% \mathrm{Cl}$ & P-value & HR & $95 \% \mathrm{Cl}$ & P-value & Coef. \\
\hline LCAT & 0.937 & $0.920-0.954$ & $<0.0001$ & 0.972 & $0.955-0.989$ & $\mathbf{0 . 0 0 1 2 4}$ & -0.028787 \\
UST & 0.94 & $0.926-0.954$ & $<0.0001$ & 0.975 & $0.958-0.993$ & $\mathbf{0 . 0 0 7 6 7}$ & -0.024918 \\
IPMK & 0.466 & $0.392-0.553$ & $<0.0001$ & & & & \\
NEU4 & 0.937 & $0.921-0.953$ & $<0.0001$ & & & & \\
\hline GALNT13 & 0.938 & $0.928-0.949$ & $<0.0001$ & 0.969 & $0.954-0.984$ & $<0.0001$ & -0.031937 \\
\hline PDE6B & 1.257 & $1.187-1.33$ & $<0.0001$ & & & & \\
\hline SMPD3 & 0.686 & $0.630-0.747$ & $<0.0001$ & 0.766 & $0.707-0.831$ & $<0.0001$ & -0.26647 \\
\hline
\end{tabular}

$\mathrm{HR}$, hazard ratio; Coef, coefficients; $\mathrm{Cl}$, confidence interval; LCAT, Lecithin-Cholesterol Acyltransferase; UST, Uronyl 2-Sulfotransferase; IPMK, Inositol Polyphosphate Multikinase; NEU4, Neuraminidase 4; GALNT13, Polypeptide N-Acetylgalactosaminyltransferase 13; PDE6B, Phosphodiesterase 6B; SMPD3, Sphingomyelin Phosphodiesterase 3; Bold text shows the P-value less than 0.05.

TABLE 3 The univariate and multivariate Cox regression analyses for clinical characteristics 


\begin{tabular}{|c|c|c|c|c|c|c|}
\hline \multirow[t]{2}{*}{ Traits } & \multicolumn{3}{|c|}{ Univariate } & \multicolumn{3}{|c|}{ Multivariate } \\
\hline & $\mathrm{HR}$ & $95 \% \mathrm{Cl}$ & $P$-value & $\mathrm{HR}$ & $95 \% \mathrm{Cl}$ & $P$-value \\
\hline Age & 5.688 & $4.262-7.591$ & $<2 \mathrm{e}-16$ *** & 2.137 & $1.511-3.021$ & $1.75 e-05 * \star *$ \\
\hline Gender & 1.113 & $0.849-1.458$ & 0.439 & - & - & - \\
\hline Grade & 5.327 & $3.699-7.673$ & $<2 e-16 * * *$ & 2.461 & $1.632-3.713$ & $1.76 \mathrm{e}-05 * \star *$ \\
\hline Risk_Score & 7.315 & $5.424-9.865$ & $<2 e-16 * * *$ & 2.840 & $1.961-4.113$ & $3.28 \mathrm{e}-08 * \star *$ \\
\hline MGMT_status & 3.326 & 2.516-4.397 & $<2 \mathrm{e}-16 * * *$ & 1.680 & $1.248-2.261$ & $0.000622 * \star *$ \\
\hline \multicolumn{7}{|c|}{$\begin{array}{l}\text { Histological subtypes } \\
\text { (Ref.=GBM) }\end{array}$} \\
\hline ASTR & 0.126 & $0.088-0.180$ & $<2 e-16 * * *$ & 0.401 & $0.265-0.607$ & $1.56 \mathrm{e}-05 * * *$ \\
\hline ODG & 0.068 & $0.044-0.103$ & $<2 e-16 * * *$ & 0.332 & $0.204-0.541$ & $9.64 \mathrm{e}-06 * \star *$ \\
\hline Mixed_glioma & 0.084 & $0.054-0.132$ & $<2 e-16 * \star *$ & 0.393 & $0.233-0.661$ & $0.000440 * * \star$ \\
\hline
\end{tabular}

MGMT, 0-(6)-methylguanine-DNA methyl transferase; GBM, glioblastoma; ASTR, astrocytoma; ODG,

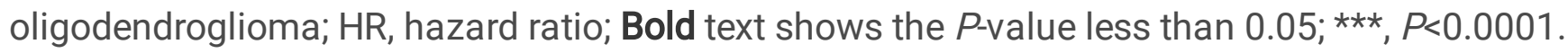

\section{Discussion}

Glioma is one of the most common malignant primary brain tumors. Generally, IDH mutation is frequently observed in grades II-III, with a frequency of $80 \%$ (45). IDH mutation confers a survival advantage for glioma patients compared to patients with the IDH wild-type (7), serving as an independent predictive indicators. Several pieces of evidence suggest that $I D H I / 2$ mutations are gain-of-function mutations, making synthetic metabolites shift from a-KG to 2-HG, which correlates with hypermethylated phenotype of glioma and propel its progression $(15,46)$. This process is opposite to IDH wild-type glioma, with catalysis of NADP+-dependent isocitrate oxidative decarboxylation to a-KG (47). However, Waitkus et al. demonstrated that an $I D H 1$ mutation $(\mathrm{R} 132 \mathrm{H}$, a commonly mutated site) reduced metabolites in the tricarboxylic acid (TCA) cycle and diminishes the growth of glioma cells (48).

As the importance of cancer stem cell indices is gradually highlighted, many studies have employed the WGCNA method to investigate the influence of mRNAsi on tumors, such as breast cancer (49), bladder cancer (50), lung squamous cell carcinoma (22), lung adenocarcinoma (51), endometrial carcinoma (52), hepatocellular carcinoma (33) and glioma (53). Nevertheless, the presence of IDH mutations in glioma makes it different from other tumors. Malta et al. demonstrated that IDH mutations in the glioma population negatively correlated with mRNAsi values (24). At the same time, metabolic alterations caused by $I D H$ mutations also in turn give rise to the dedifferentiation of glioma cells. Due to these dedifferentiation and metabolic changes conferring $\mathrm{IDH}$-mutant glioma patients a better prognosis than IDH wild-type glioma patients. However, pathogenesis of gliomas remains unclear. Accordingly, in the 
present study, we used GBM and LGG samples from the TCGA database, with corresponding mRNAsi values of each sample, to explore the mRNAsi relationship to clinical characteristics, especially in terms of IDH status. Next, WGCNA was employed to identify the genes in each module associated with the mRNAsi trait, and cancer stem cell-related metabolic genes were determined after identification of metabolism-related genes from 70 KEGG pathways. Ultimately, a four-gene signature was constructed via univariate and multivariate Cox regression analyses, and this signature was verified using internal (TCGA cohort) and external datasets (CGGA validation set). Our results showed that the four-gene signature (LCAT, UST, GALNT13 and SMPD3 genes) was independent indicator for the prediction of glioma survival. We also found that the four genes were protective factors for glioma patients, with high expression in IDH mutant glioma samples.

LCAT (Lecithin-Cholesterol Acyltransferase) encodes an extracellular cholesterol esterifying enzyme, a lecithin-cholesterol acyltransferase. The esterification of cholesterol plays a pivotal functional role in transporting cholesterol (54). Given that lipid metabolism is also involved in metabolic reprogramming, $\angle C A T$ may serve as an important prognostic biomarker. A previous study, Ouyang et al., constructed a twelve-gene signature, including the SPP1, KIF20A, HMMR, TPX2, LAPTM4B, TTK, MAGEA6, ANX10, $\angle E C T 2, C Y P 2 C 9, R D H 16$ and $\angle C A T$ genes, for predicting the OS in hepatocellular carcinoma (HCC). That study also indicated that LCAT was expressed at a low level in HCC patients (55). Additionally, UST (Uronyl 2-Sulfotransferase), located on chromosome 6q25.1, encodes a protein that transfers sulfate to the 2 site of uronyl residues, including iduronyl residues and glucuronyl residues (56). Nikolovska et al. recently demonstrated that UST participates in melanoma cell migration, and knock-down of the UST gene suppressed cell motility and adhesion (57), which may relate to glycosaminoglycan metabolism, but the specific mechanism is unclear. The absence of studies about the association between the UST gene and glioma to date encourages us to investigate this question in the future. The GALNT13 (Polypeptide NAcetylgalactosaminyltransferase 13) gene encodes the GALNT13 protein, which catalyzes the initial reaction in O-linked oligosaccharide biosynthesis, holding the possibility of transferring an N-acetyl-Dgalactosamine residue to a serine or threonine residue on the protein receptor, and is the pivotal enzyme involved in the synthesis of O-glycans, especially Tn epitopes in neurons (58). Ducray et al. used real-time RT PCR to detect 22 DEGs between 1p19q co-deleted and epidermal growth factor receptor (EGFR) amplified gliomas and found that GALNT13 was down-regulated in glioma patients with EGFR amplification compared with normal brain samples (59). Additionally, the findings in our study denote that a low expression level of GALNT13 is correlated with unfavorable outcome in glioma patients. Meanwhile, the results of Nogimori et al. also demonstrated that GALNT13 was a the prognostic factor for lung cancer (60). The protein encoded by the SMPD3 (Sphingomyelin Phosphodiesterase 3) gene, mainly expressed in neurons of the central nervous system (CNS), catalyzes the hydrolysis process of sphingomyelin to generate phosphocholine andceramide (61), which mediates a wide range of cellular functions and is able to independently modulate cell growth, differentiation, cell cycle and its apoptosis (62). The occurrence of mutations in SMPD3 may be conducive to leukemia formation (63). SMPD3, functions as a tumor suppressor gene in $\mathrm{HCC}$ via integrative genomic analysis and affects tumor invasion. Decreased SMPD3 is an independent predictive factor influencing the early recurrence of HCC 
(64). The above mentioned evidence suggests that these four genes may be the prognostic indicators of tumors, but the specific mechanism needs to be explored.

The present study provides insights into the impact of cancer stem cell (CSC)-associated metabolic genes on glioma survival. To our knowledge, metabolic reprogramming of CSC may be characterized by carcinogenic activity (65-67). A number of previous studies have shown metabolic alterations in IDH mutant glioma. Accordingly, we have identified stemness-related metabolic genes and established a four gene-based prognostic model, which raises hope for clinical use.

However, some limitations also need to be mentioned. First, we used data from public databases to perform analysis, and lack experimental verification of gene expression and exploration of the specific mechanisms and pathways. Furthermore, our laboratory also lacked the experimental condition for inhibiting the genes or knocking down them to confirm whether these four hub genes can be mechanistically targeted to reduce glioma malignancy. Thus, the lack of experimental verification is our study's major limitations. Besides, the expression of $L C A T$ protein between normal brain and glioma tissues was unaccessible in the HPA database. However, the tissues of glioma patients also hardly to collect in clinic for verifying its expressions, which needs the cooperation of neurosurgeons in the near future. Also, other molecular alterations, such as TERT promoter mutations, ATRX mutations or loss and co-deletion of chromosome 1p/19q may exert impact on gliomas' prognosis. Due to lack of sufficient information on the molecular characteristics in the assessed glioma samples, we did not take these factors into consideration. Ultimately, multi-center prospective studies will be required for evaluation the clinical application of the four gene prognostic signature.

\section{Conclusions}

Taken together, a prognostic four gene signature was constructed via integrative bioinformatics analysis combining the TCGA and CGGA cohorts. This prognostic model effectively stratified glioma patients and was found to be an independent factor for predicting gliomas' OS. Our findings may provide insight into individualized tumor therapies in the context of clinical settings. Thus, we recommended that this classifier should be employed to assess the prognostic risk of glioma patients. And a multitude of prospective studies will be needed to verify our findings.

\section{Declarations}

\section{AUTHOR CONTRIBUTIONS}

YT, YJ and YG contributed to the planning of the study. YT and $Y J$ drafted the manuscript. $H T$ and $Y G$ revised the manuscript. DZ, JF, ZZ and YL downloaded the data. YT, YJ and YG established the four gene signature. $Y T, Y J, J Y, Z Z$ and $H T$ made contributions to validation of the prognostic model. $Y T$ and $Y J$ prepared all tables and figures. YT, DZ, JF, JY, HT and YG preformed the interpretation of all results. All the authors reviewed and approved the final manuscript- 


\section{DECLARATION OF INTERESTS}

The authors declare that they have no competing interests.

\section{ACKNOWLEDGMENTS}

The authors thank Yi Liao, PhD, who provided the R code for WGCNA. We thank International Science Editing (http://www.internationalscienceediting.com) for editing this manuscript.

\section{DATA AVAILABILITY STATEMENT}

The TCGA-GBM, TCGA-LGG (https://portal.gdc.cancer.gov); mRNAsi and EREG-mRNAsi (https://www.ncbi.nlm.nih.gov/pmc/articles/PMC5902191/); clinical information of TCGA samples (www.cbioportal.org); mRNA Sequencing data of CGGA_mRNAseq_693 and CGGA_mRNAseq_325 (www.cgga.org.cn/); metabolic genes lists (https://figshare.com/s/d2a968666f6f4c9ea224 ); The human protein atlas database (HPA, https://www.proteinatlas.org/).

\section{FUNDING}

This research was supported by the Youth Fund of Southwest Medical University (no. 2018-ZRQN-125).

\section{References}

1. Ostrom Q, Cioffi G, Gittleman H, Kruchko C, Waite K, Barnholtz-Sloan J (2019) CBTRUS Statistical Report: Primary Brain and Other Central Nervous System Tumors Diagnosed in the United States in 2012-2016. Neuro Oncol 21:v1-100. https://doi.org/10.1093/neuonc/noz150

2. Behin A, Hoangxuan K, Carpentier AF, Delattre JY (2018) Primary brain tumours in adults. Lancet 392:432 - 46.https:// doi.org/ 10.1016/S0140-6736(18)30990-5

3. Ostrom Q, Gittleman H, Liao P, Vecchione-Koval T, Wolinsky Y, Kruchko C et al (2017) CBTRUS Statistical Report: Primary brain and other central nervous system tumors diagnosed in the United States in 2010-2014. Neuro Oncol 19:v1-88.https:// doi.org/10.1093/neuonc/nox158

4. Louis DN, Ohgaki H, Wiestler OD, Cavenee WK, Burger PC, Jouvet A et al (2007) The 2007 WHO Classification of Tumours of the Central Nervous System. Acta Neuropathol 114:97-109. https://doi.org/10.1007/s00401-007-0243-4

5. National Health Commission Medical Administration and Hospital Administration. Guidelines for the diagnosis and treatment of glioma (2018 edition). Chin J Neurosurg. (2019) 35:217-239. https://doi.org/10.3760 / cma.j.issn. 1001-2346.2019.03.001

6. Jiang T, Mao Y, Ma W, Mao Q, You Y, Yang X et al (2016) CGCG clinical practice guidelines for the management of adult diffuse gliomas. Cancer Lett 375:263-273. https://doi.org/10.1016/ j. canlet.2016.01.024 
7. Schiff D, Van den Bent M, Vogelbaum MA, Wick W, Miller CR, Taphoorn M et al (2019) Recent Developments and Future Directions in Adult Lower-Grade Gliomas: Society for Neuro-Oncology (SNO) and European Association of Neuro-Oncology (EANO) Consensus. Neuro Oncology 21:837853. https://doi.org/10.1093/neuonc/noz033

8. Zhang CB, Bao ZS, Wang HJ, Yan W, Liu YW, Li MY et al (2014) Correlation of IDH1/2 mutation with clinicopathologic factors and prognosis in anaplastic gliomas: a report of 203 patients from China. $J$ Cancer Res Clin Oncol 140:45-51.https://doi.org/10.1007/ s00432-013-1519-9

9. Cai J, Yang P, Zhang W, Liu Y, Bao Z, Liu X et al (2014) ATRX mRNA expression combined with IDH1/2 mutational status and Ki-67 expression refines the molecular classification of astrocytic tumors: evidence from the whole transcriptome sequencing of 169 samples. Oncotarget 5:25512561. https://doi.org/10.18632/oncotarget.1838

10. Pavlova NN, Thompson CB (2016) The Emerging Hallmarks of Cancer Metabolism. Cell Metab 23:27-47. .https://doi.org/10.1016/j.cmet.2015.12.006

11. Reitman ZJ, Duncan CG, Poteet E, Winters A, Yan LJ, Gooden DM et al (2014) Cancer-associated isocitrate dehydrogenase 1 (IDH1) R132H mutation and d-2-hydroxyglutarate stimulate glutamine metabolism under hypoxia. J Biol Chem 289:23318-23328. https://doi.org/ 10.1074/ jbc.M114.575183

12. Chen R, Nishimura MC, Kharbanda S, Peale F, Deng Y, Daemen et al (2014) Hominoid-specific enzyme GLUD2 promotes growth of IDH1R132H glioma. Proc Natl Acad Sci U S A 111:1421714222. https://doi.org/10.1073/pnas.1409653111

13. Ohka F, Ito M, Ranjit M, Senga T, Motomura A, Motomura K et al (2014) Quantitative metabolome analysis profiles activation of glutaminolysis in glioma with IDH1 mutation. Tumour Biol 35:59115920. https://doi.org/10.1007/s13277-014-1784-5

14. Duncan CG, Barwick BG, Jin G, Rago C, Kapoor-Vazirani P, Powell DR et al (2012) A heterozygous IDH1R132H/WT mutation induces genome-wide alterations in DNA methylation. Genome Res 22:2339-2355. https://doi.org/10.1101/gr.132738.111

15. Turcan S, Rohle D, Goenka A, Walsh LA, Fang F, Yilmaz E et al (2012) IDH1 mutation is sufficient to establish the glioma hypermethylator phenotype. Nature 483:479-483. https://doi.org/10.1038/nature10866

16. Lu C, Ward PS, Kapoor Gs, Rohle D, Turcan S, Abdel-Wahab O et al (2012) IDH mutation impairs histone demethylation and results in a block to cell differentiation. Nature 483:474-478. https://doi.org/10.1038/nature10860

17. Spencer DA, Auffinger BM, Murphy JP, Muroski ME, Qiao J, Gorind Y et al (2017) Hitting a Moving Target: Glioma Stem Cells Demand New Approaches in Glioblastoma Therapy. Curr Cancer Drug Targets 17:236-254. https://doi.org/10.2174/1568009616666161215161924

18. Friedmann-Morvinski D, Verma IM (2014) Dedifferentiation and reprogramming: origins of cancer stem cells. Embo Rep 15:244-253. https://doi.org/10.1002/embr.201338254 
19. Visvader JE, Lindeman GJ (2012) Cancer stem cells: current status and evolving complexities. Cell Stem Cell 10:717-728. https://doi.org/10.1016/j.stem.2012.05.007

20. Easwaran H, Tsai HC, Baylin SB (2014) Cancer epigenetics: tumor heterogeneity, plasticity of stemlike states, and drug resistance. Mol Cell. 54:716 - 27. https://doi.org/10.1016/j.molcel. 2014. 05.015

21. Sokolov A, Paull EO, Stuart JM (2016) One-class detection of cell states in tumor subtypes. Pac Symp Biocomput 21:405-416

22. Liao Y, Xiao H, Cheng M, Fan X (2020) Bioinformatics Analysis Reveals Biomarkers With Cancer Stem Cell Characteristics in Lung Squamous Cell Carcinoma. Front Genet 11:427. https://doi.org/10.3389/fgene.2020.00427

23. Pei S, Chen L, Yang Y, Zhu X (2020) Identification of genes associated with cancer stem cell characteristics in head and neck squamous cell carcinoma through co-expression network analysis. Head Neck 42:2460-2472. https://doi.org/10.1002/hed.26266

24. Malta TM, Sokolov A, Gentles AJ, Burzykowski T, Poisson L, Weinstein JN et al (2018) Machine Learning Identifies Stemness Features Associated with Oncogenic Dedifferentiation. Cell 173:338354. .https://doi.org/10.1016/j.cell.2018.03.034

25. Wang Y, Qian T, You G, Peng X, Chen C, You Y et al (2015) Localizing seizure-susceptible brain regions associated with low-grade gliomas using voxel-based lesion-symptom mapping. Neuro Oncol 17:282-288. https://doi.org/10.1093/neuonc/nou130

26. Liu X, Li Y, Qian Z, Sun Z, Xu K, Wang K et al (2018) A radiomic signature as a non-invasive predictor of progression-free survival in patients with lower-grade gliomas. Neuroimage Clin 20:1070-1077. https://doi.org/10.1016/j.nicl.2018.10.014

27. Bao ZS, Chen HM, Yang MY, Zhang CB, Yu K, Ye WL et al (2014) RNA-seq of 272 gliomas revealed a novel, recurrent PTPRZ1-MET fusion transcript in secondary glioblastomas. Genome Res 24:17651773. https://doi.org/10.1101/gr.165126.113

28. Zhao Z, Meng F, Wang W, Wang Z, Zhang C, Jiang T (2017) Comprehensive RNA-seq transcriptomic profiling in the malignant progression of gliomas. Sci Data 4:170024. https://doi.org/ 10.1038/sdata.2017.24

29. Leek JT, Johnson WE, Parker HS, Jaffe AE, Storey JD (2012) The sva package for removing batch effects and other unwanted variation in high-throughput experiments. Bioinformatics 28:882-883. https://doi.org/10.1093/bioinformatics/bts034

30. Camp RL, Dolled-Filhart M, Rimm DL (2004) X-tile: a new bio-informatics tool for biomarker assessment and outcome-based cut-point optimization. Clin Cancer Res 10:7252-7259. https://doi.org/10.1158/1078-0432.CCR-04-0713

31. Langfelder P, Horvath S (2008) WGCNA: an R package for weighted correlation network analysis. Bmc Bioinformatics 9:559. https://doi.org/10.1186/1471-2105-9-559

32. Sun X, Shang J, Wu A, Xia J, Xu F (2020) Identification of dynamic signatures associated with smoking-related squamous cell lung cancer and chronic obstructive pulmonary disease. J Cell Mol 
Med 24:1614-1625. https://doi.org/10.1111/jcmm.14852

33. Bai KH, He SY, Shu LL, Wang WD, Lin SY, Zhang QY et al (2020) Identification of cancer stem cell characteristics in liver hepatocellular carcinoma by WGCNA analysis of transcriptome stemness index. Cancer Med-Us 9:4290-4298. https://doi.org/10.1002/cam4.3047

34. Yu G, Wang L, Han Y, He QY (2012) clusterProfiler: an R Package for Comparing Biological Themes Among Gene Clusters. Omics 16:284-287. https://doi.org/10.1089/omi.2011.0118

35. Ma B, Jiang H, Wen D, Hu J, Han L, Liu W et al (2019) Transcriptome analyses identify a metabolic gene signature indicative of dedifferentiation of papillary thyroid cancer. J Clin Endocr Metab 104:3713-3725. https://doi.org/10.1210/jc.2018-0268

36. Yamaoka K, Nakagawa T, Uno T (1978) Application of akaike's information criterion (AIC) in the evaluation of linear pharmacokinetic. J Pharmacokinet Biopharm 6:165-175. https://doi.org/10.1007/bf01117450

37. She $Y$, Kong X, Ge Y, Yin P, Liu Z, Chen J et al (2020) Immune-related gene signature for predicting the prognosis of head and neck squamous cell carcinoma. Cancer Cell Int 20:22. https://doi.org/10.1186/s12935-020-1104-7

38. Heagerty PJ, Zheng Y (2005) Survival model predictive accuracy and ROC curves. Biometrics 61:92105. https://doi.org/10.1111/j.0006-341X.2005.030814.x

39. Yan G, Greene $T$ (2008) Investigating the effects of ties on measures of concordance. Stat Med 27:4190-4206. https://doi.org/10.1002/sim.3257

40. Frank EAHJ. RMS: Regression Modeling Strategies.(2019) R Package Version 3

41. Huang R, Chen Z, Li W, Fan C, Liu J (2020) Immune system-associated genes increase malignant progression and can be used to predict clinical outcome in patients with hepatocellular carcinoma. Int J Oncol 56:1199-1211. https://doi.org/10.3892/ijo.2020.4998

42. Iasonos A, Schrag D, Raj GV, Panageas KS (2008) How To Build and Interpret a Nomogram for Cancer Prognosis. J Clin Oncol 26:1364-1370. https://doi.org/10.1200/JC0.2007.12.9791

43. Uhlén M, Fagerberg L, Hallström BM, Lindskog C, Oksvold P, Mardinoglu A et al (2015) Proteomics. Tissue-based map of the human proteome. Science 347:1260419.https://doi.org/ 10.1126/science. 1260419

44. Zhang M, Wang X, Chen X, Guo F, Hong J (2020) Prognostic Value of a Stemness Index-Associated Signature in Primary Lower-Grade Glioma. Front Genet 11:441. https://doi.org/10.3389/fgene.2020.00441

45. Yan H, Parsons DW, Jin G, McLendon R, Rasheed BA, Yuan W et al (2009) IDH1 and IDH2 Mutations in Gliomas. N Engl J Med 360:765-773. https://doi.org/10.1056/NEJMoa0808710

46. Noushmehr H, Weisenberger DJ, Diefes K, Phillips HS, Pujara K, Berman BP et al (2010) Identification of a $\mathrm{CpG}$ island methylator phenotype that defines a distinct subgroup of glioma. Cancer Cell 17:510-522. https://doi.org/10.1016/j.ccr.2010.03.017 
47. Dang L, White DW, Gross S, Bennett BD, Bittinger MA, Driggers EM et al (2009) Cancer-associated IDH1 mutations produce 2-hydroxyglutarate. Nature 462:739-744. https://doi.org/

$10.1038 /$ nature08617

48. Waitkus MS, Pirozzi CJ, Moure CJ, Diplas BH, Hansen LJ, Carpenter AB et al (2018) Adaptive evolution of the GDH2 allosteric domain promotes gliomagenesis by resolving IDH1R132H induced metabolic liabilities. Cancer Res 78:36-50. https://doi.org/10.1158/ 0008-5472. CAN- 17-1352

49. Pei J, Wang Y, Li Y (2020) Identification of key genes controlling breast cancer stem cell characteristics via stemness indices analysis. J Transl Med 18:74.https://doi.org/10.1186/ s12967020-02260

50. Pan S, Zhan Y, Chen X, Wu B, Liu B (2019) Identification of Biomarkers for Controlling Cancer Stem Cell Characteristics in Bladder Cancer by Network Analysis of Transcriptome Data Stemness Indices. Front Oncol 9:613. https://doi.org/10.3389/fonc.2019.00613

51. Liao Y, Wang Y, Cheng M, Huang C, Fan X (2020) Weighted Gene Coexpression Network Analysis of Features That Control Cancer Stem Cells Reveals Prognostic Biomarkers in Lung Adenocarcinoma. Front Genet 11:311. https://doi.org/10.3389/fgene.2020.00311

52. Liu J, Wu Z, Sun R, Nie S, Meng H, Zhong Y et al (2020) Using mRNAsi to identify prognostic-related genes in endometrial carcinoma based on WGCNA. Life Sci 258:118231. https://doi.org/10.1016/j.Ifs.2020.118231

53. Xia P, Li Q, Wu G, Huang Y (2020) Identification of Glioma Cancer Stem Cell Characteristics Based on Weighted Gene Prognosis Module Co-Expression Network Analysis of Transcriptome Data Stemness Indices. J Mol Neurosci. https://doi.org/10.1007/s12031-020-01590-Z

54. Clay MA, Pyle DH, Rye KA, Barter PJ.(2000) Formation of Spherical, Reconstituted High Density Lipoproteins Containing Both Apolipoproteins A-I and A-II Is Mediated by Lecithin:Cholesterol Acyltransferase. J Biol Chem 275:9019-9025. https://doi.org/ 10.1074/jbc. 275.12.9019

55. Ouyang G, Yi B, Pan G, Chen X (2020) A robust twelve-gene signature for prognosis prediction of hepatocellular carcinoma. Cancer Cell Int 20:207. https://doi.org/10.1186/ s12935-020-01294-9

56. Kobayashi M, Sugumaran G, Liu J, Shworak NW, Silbert JE, Rosenberg RD (1999) Molecular cloning and characterization of a human uronyl 2-sulfotransferase that sulfates iduronyl and glucuronyl residues in dermatan/chondroitin sulfate. J Biol Chem 274:10474-10480.https://doi.org/ 10.1074/jbc.274.15.10474

57. Nikolovska K, Spillmann D, Haier J, Ladányi A, Stock C, Seidler DG (2017) Melanoma Cell Adhesion and Migration Is Modulated by the Uronyl 2-O Sulfotransferase. Plos One 12:e170054. https://doi.org/10.1371/journal.pone.0170054

58. Zhang Y, Iwasaki H, Wang H, Kudo T, Kalka TB, Hennet T et al (2003) Cloning and characterization of a new human UDP-N-acetyl-alpha-D-galactosamine: polypeptide $\mathrm{N}$-acetylgalactosaminyltransferase, designated pp-GaINAc-T13, that is specifically expressed in neurons and synthesizes GaINAc alphaserine/threonine antigen. J Biol Chem 278:573-584. https://doi.org/10.1074/jbc.M203094200 
59. Ducray F, Idbaih A, de Reyniès A, Bièche I, Thillet J, Mokhtari K et al (2008) Anaplastic oligodendrogliomas with 1p19q codeletion have a proneural gene expression profile. Mol Cancer 7:41. https://doi.org/10.1186/1476-4598-7-41

60. Nogimori K, Hori T, Kawaguchi K, Fukui T, Mii S, Nakada H et al (2016) Increased expression levels of ppGalNAc-T13 in lung cancers: Significance in the prognostic diagnosis. Int J Oncol 49:1369-1376. https://doi.org/10.3892/ijo.2016.3638

61. Stoffel W, Jenke B, Block B, Zumbansen M, Koebke J (2005) Neutral sphingomyelinase 2 (smpd3) in the control of postnatal growth and development. Proc Natl Acad Sci U S A 102:4554-4559. https://doi.org/10.1073/pnas.0406380102

62. Stoffel W, Jenke B, Holz B, Binczek E, Günter RH, Knifka J et al (2007) Neutral sphingomyelinase (SMPD3) deficiency causes a novel form of chondrodysplasia and dwarfism that is rescued by Col2A1-driven smpd3 transgene expression. Am J Pathol 171:153-161. https://doi.org/10.2353/ajpath.2007.061285

63. Kim WJ, Okimoto RA, Purton LE, Goodwin M, Haserlat SM, Dayyani F et al (2008) Mutations in the neutral sphingomyelinase gene SMPD3 implicate the ceramide pathway in human leukemias. Blood 111:4716-4722. https://doi.org/10.1182/blood-2007-10-113068

64. Revill K, Wang T, Lachenmayer A, Kojima K, Harrington A, Li J et al (2013) Genome-wide methylation analysis and epigenetic unmasking identify tumor suppressor genes in hepatocellular carcinoma. Gastroenterology 145:1424-1435. https://doi.org/10.1053/j.gastro.2013.08.055

65. Menendez JA, Vellon L, Oliveras-Ferraros C, Cufí S, Vazquez-Martin A (2011) mTOR-regulated senescence and autophagy during reprogramming of somatic cells to pluripotency: a roadmap from energy metabolism to stem cell renewal and aging. Cell Cycle 10:3658-3677. https://doi.org/10.4161/cc.10.21.18128

66. Vazquez-Martin A, Vellon L, Quirós P, Cufí S, Ruiz de Galarreta E, Oliveras-Ferraros C et al (2012) Activation of AMP-activated protein kinase (AMPK) provides a metabolic barrier to reprogramming somatic cells into stem cells. Cell Cycle.11:974 - 89.https://doi.org/ 10.4161/ cc. 11.5.19450

67. Vazquez-Martin A, Corominas-Faja B, Cufi S, Vellon L, Oliveras-Ferraros C, Menendez OJ et al (2013) The mitochondrial $\mathrm{H}(+)$-ATP synthase and the lipogenic switch: new core components of metabolic reprogramming in induced pluripotent stem (iPS) cells. Cell Cycle 12:207-218. https://doi.org/10.4161/cc.23352

\section{Figures}



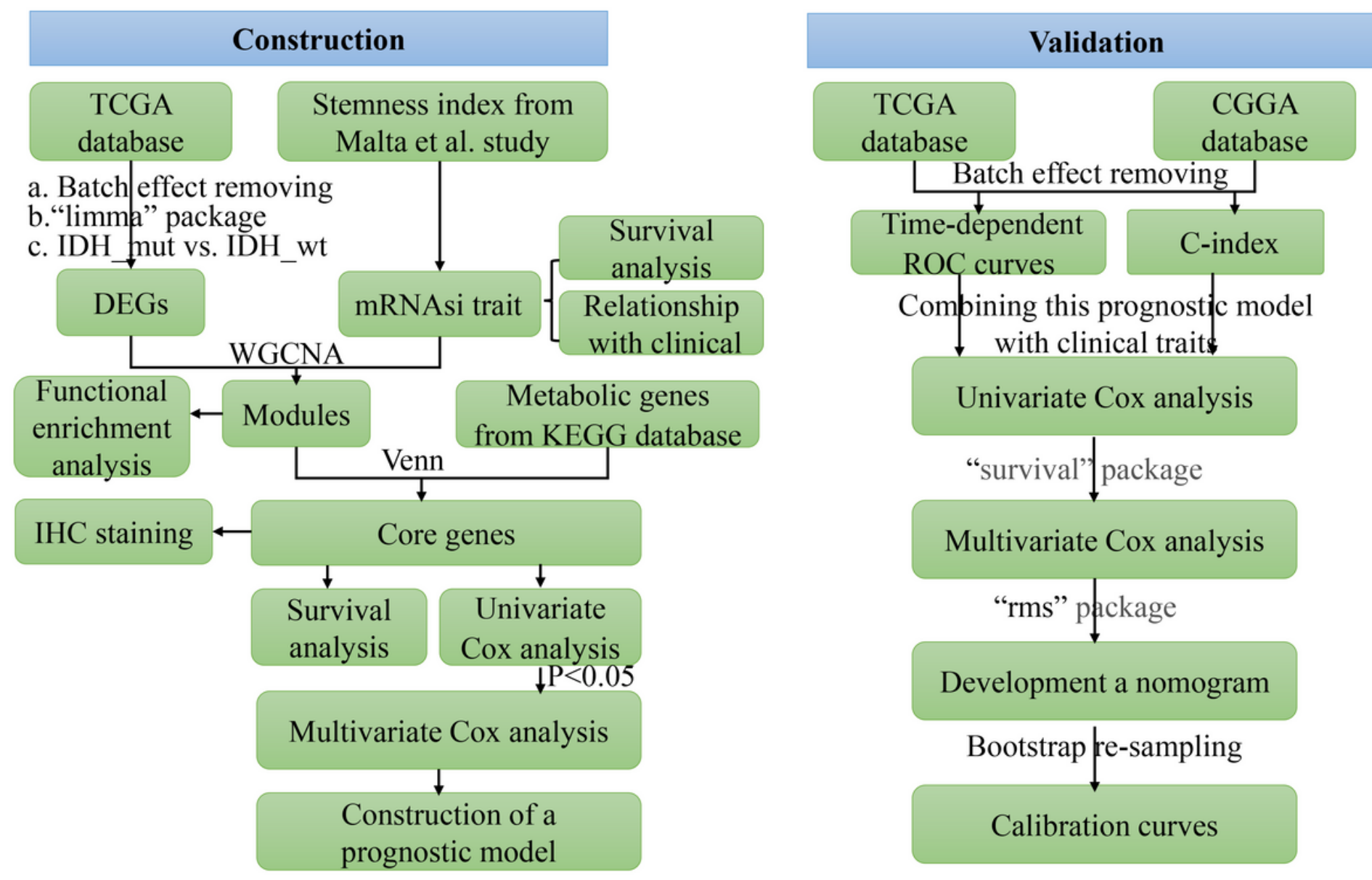

a. Batch effect removing
b."limma" package
c. IDH_mut vs. IDH_wt
Batch effect removing

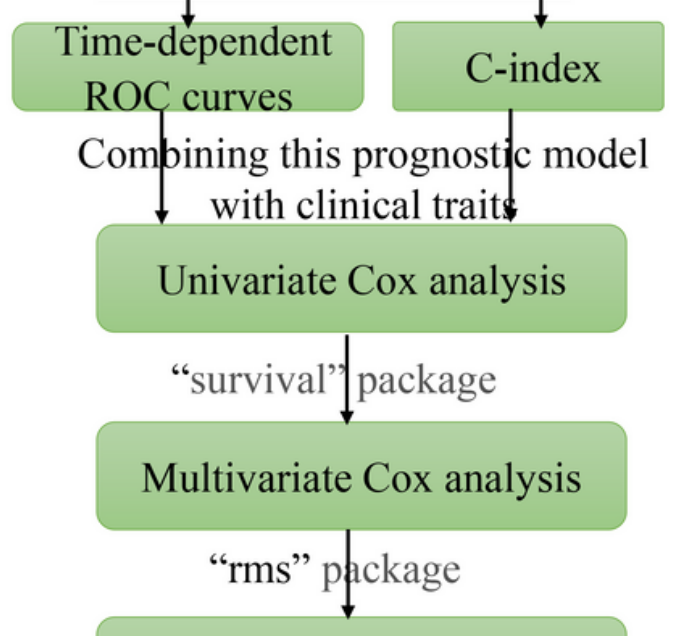

Development a nomogram

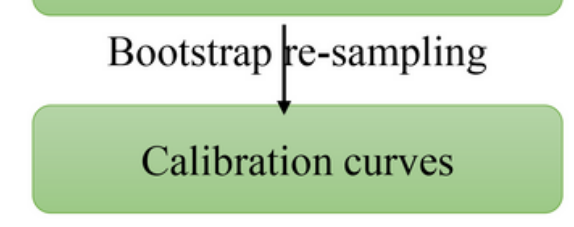

\section{Figure 1}

Flow chart of the present study. TCGA, The Cancer Genome Atlas; mRNAsi, gene expression-based stemness indices; IDH, isocitrate dehydrogenase; WGCNA, weighted gene correlation network analysis; IHC, immunochemistry; CGGA, the Chinese Glioma Genome Atlas; C-index, concordance index; ROC, receiver operating characteristics. 
A

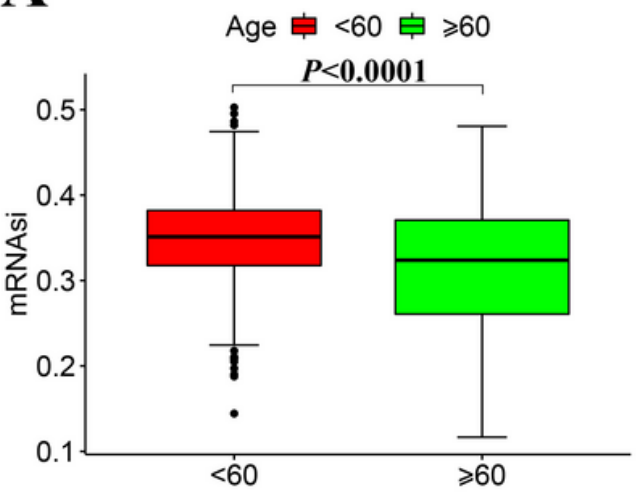

D

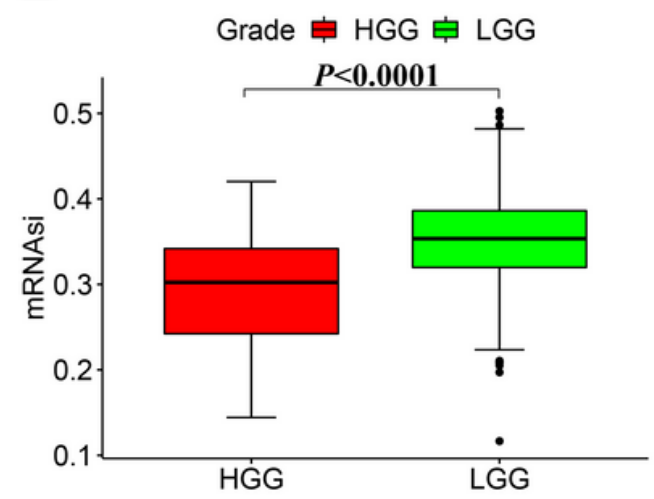

B

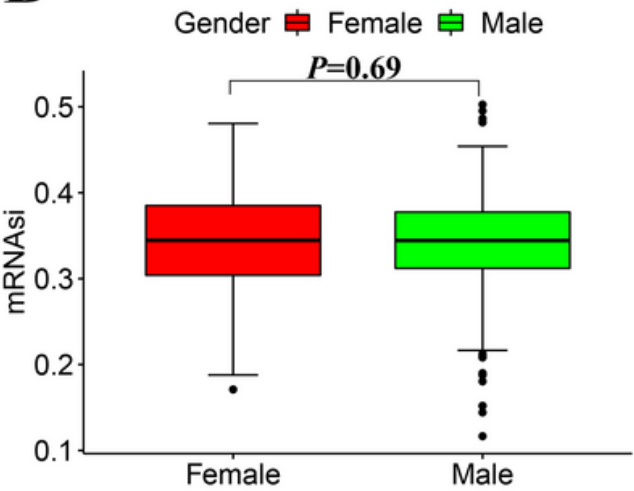

$\mathbf{E}$

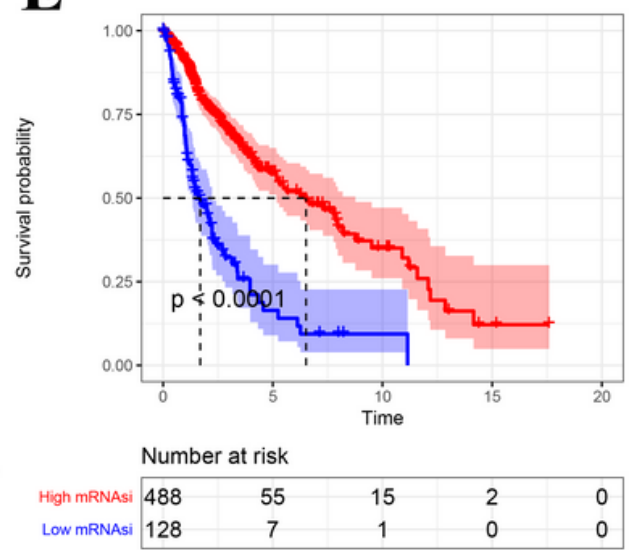

C

IDH_status 追 IDH_mut 帛 IDH_wt $P<0.0001$

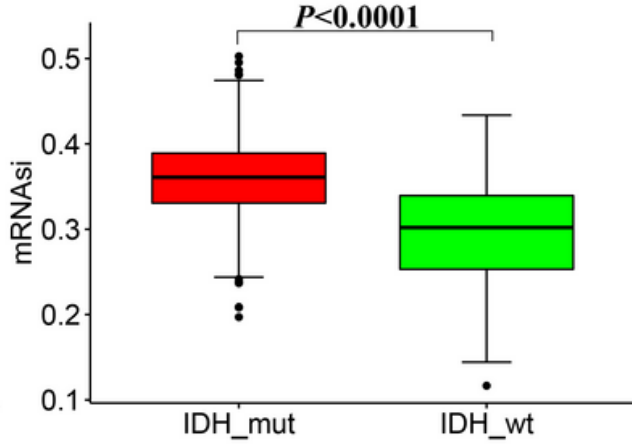

W EREGmRnAsi + High EREGmRnAsi - Low EREGmRNAsi

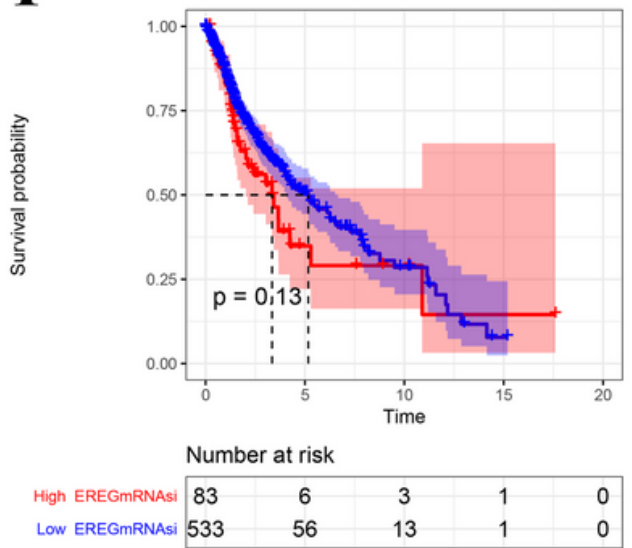

Figure 2

mRNAsi in relation to clinical traits of glioma patients. (A) Boxplot of mRNAsi in glioma samples, stratified by age; (B) boxplot of mRNAsi in glioma patients, grouped by gender; (C) boxplot of mRNAsi in glioma patients based on IDH status; (D) boxplot of mRNAsi in glioma patients on the basis of tumor grade; (E) KM survival curves displaying the overall survival of glioma patients divided into high or low mRNAsi groups based on the optimal cut-off values calculated by X-tile software; (F) KM survival curves showed the overall survival of glioma patients with high or low-ERGE-mRNAsi values. mRNAsi, gene expression-based stemness indices; IDH, isocitrate dehydrogenase; IDH_mut, IDH mutation; IDH_wt, IDH wild type; ERGE-mRNAsi, epigenetically regulated-mRNAsi; LGG, low-grade glioma; HGG, high-grade glioma; KM, Kaplan-Meier. 

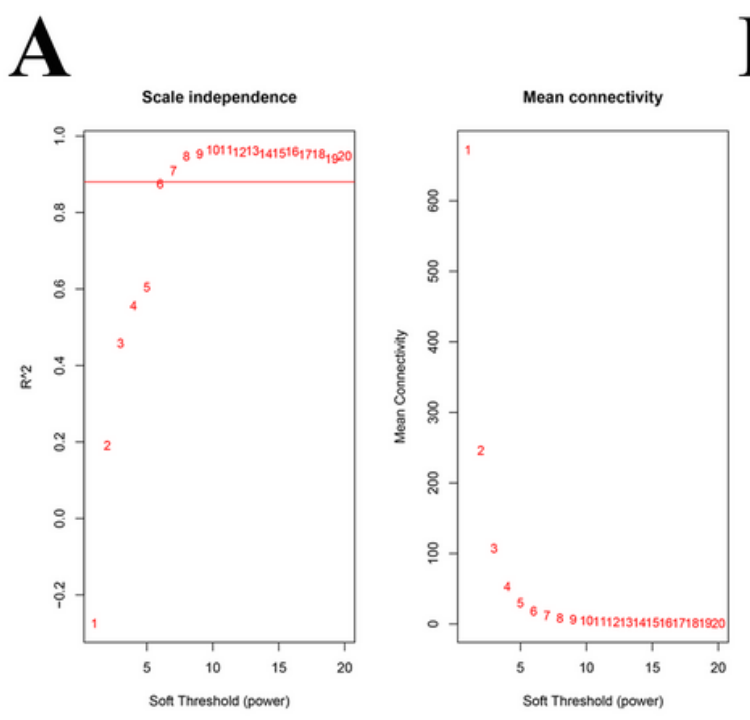

B
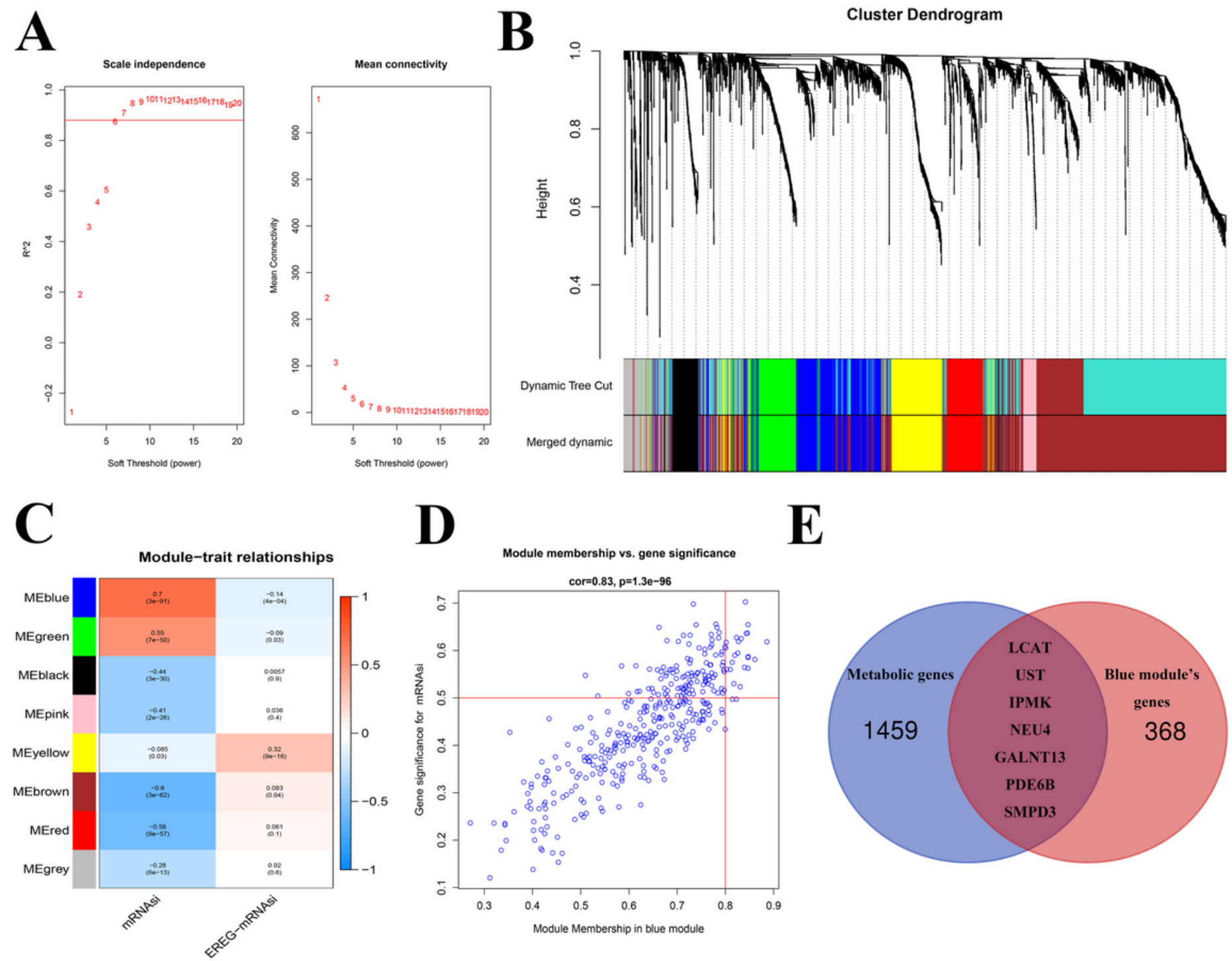

\section{$\mathbf{E}$}

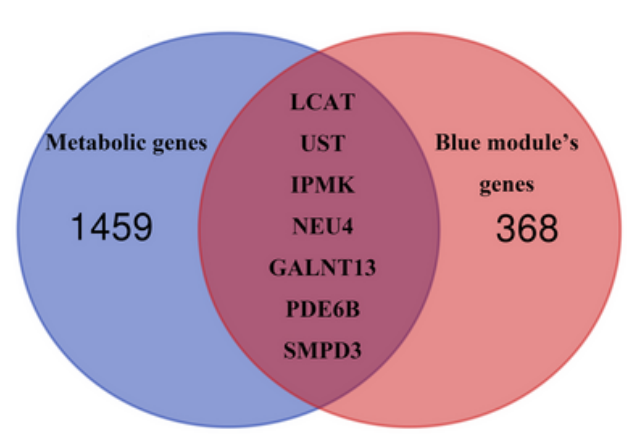

Figure 3

WGCNA and identification of core genes. (A) The network topology of a variety of soft threshold powers $(\beta)$; The left panel presents the scale-free fit index, with the $y$-axis showing $\mathrm{R}^{\wedge} 2$ and the $x$-axis showing the soft threshold powers, where six was selected as the appropriate power value for next analysis. The right panel denotes that mean connectivity ( $y$-axis) gradually decreases as the $\beta$ values (x-axis) increase; (B) Cluster dendrogram of glioma patients. Each branch is representative of a gene and the each color is a co-expression module; (C) Correlation between the modules and clinical traits (mRNAsi and EREGmRNAsi). The correlation coefficient of each cell refers to the correlation of gene modules in relation to clinical characteristics, with its the size increasing from blue to red; (D) Scatter plot for module membership (MM) vs. gene significance (GS) for mRNAsi in the blue module; (E) Identification of seven core genes via a Venn plot. WGCNA, weighted gene correlation network analysis; mRNAsi, gene expression-based stemness indices; ERGE-mRNAsi, epigenetically regulated-mRNAsi; KEGG, Kyoto Encyclopedia of Genes and Genomes. 
A

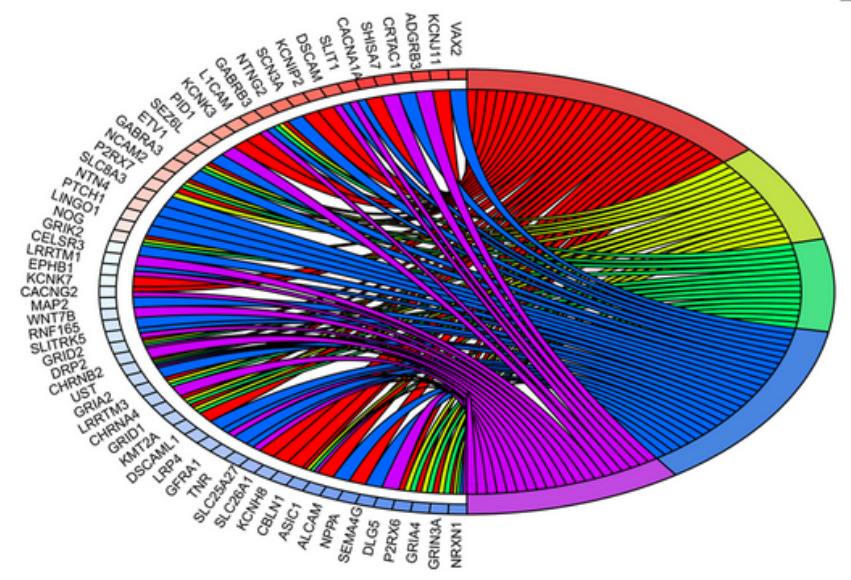

GO\&BP

Regulation of membrane potential $\square$ Regulation of postsynaptic membrane potential $\square$ Chemical synaptic transmission, postsynaptic

$\square$ Synapse organization
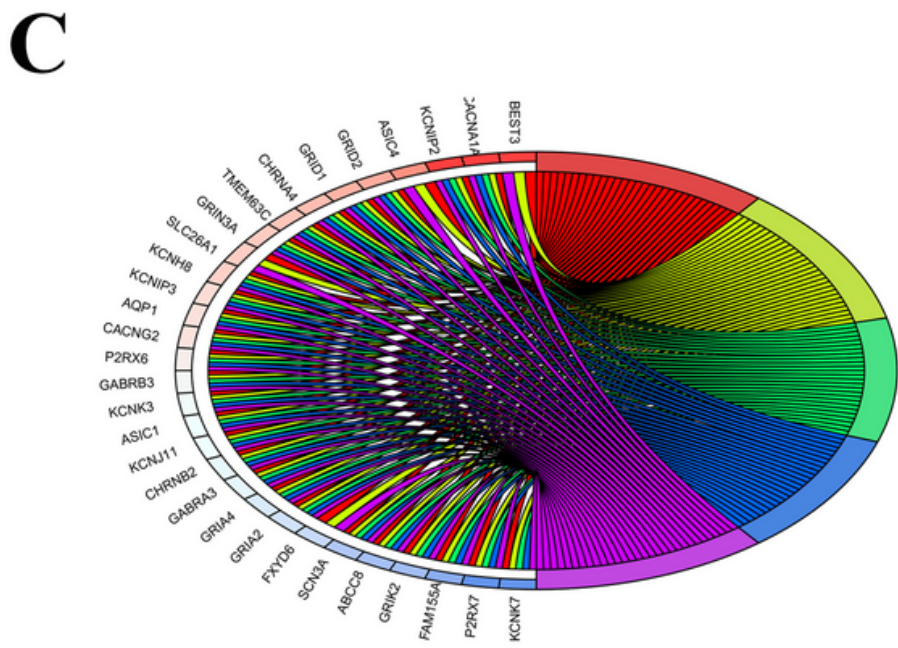

GO\&MF

$\square$ Ion channel activity $\square$ Substrate-specific channel activity $\square$ Ion gated channel activity $\square$ Gated channel activity $\square$ Channel activity
B

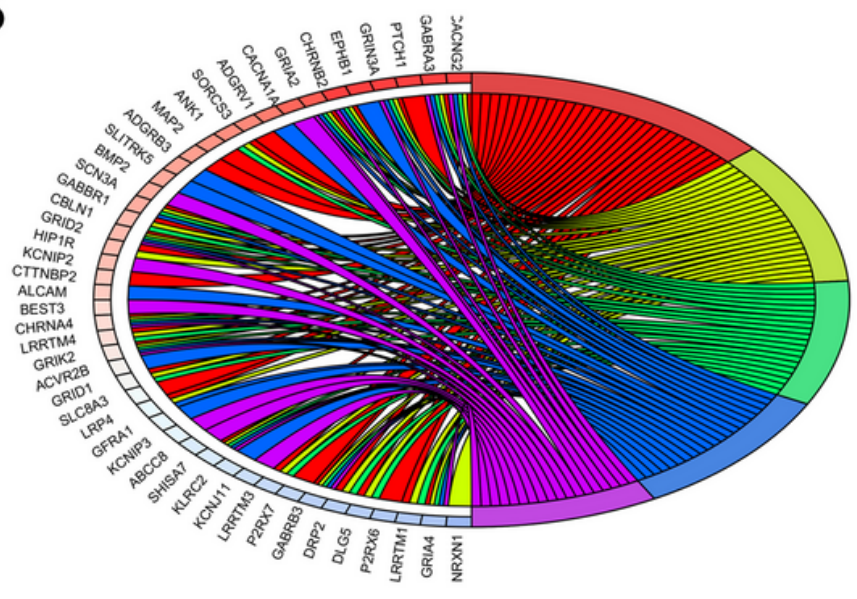

GO\&CC

Postsynapse $\square$ Synaptic membrane $\square$ Postsynaptic membrane

Receptor complex $\square$ Ion channel complex

\section{D}

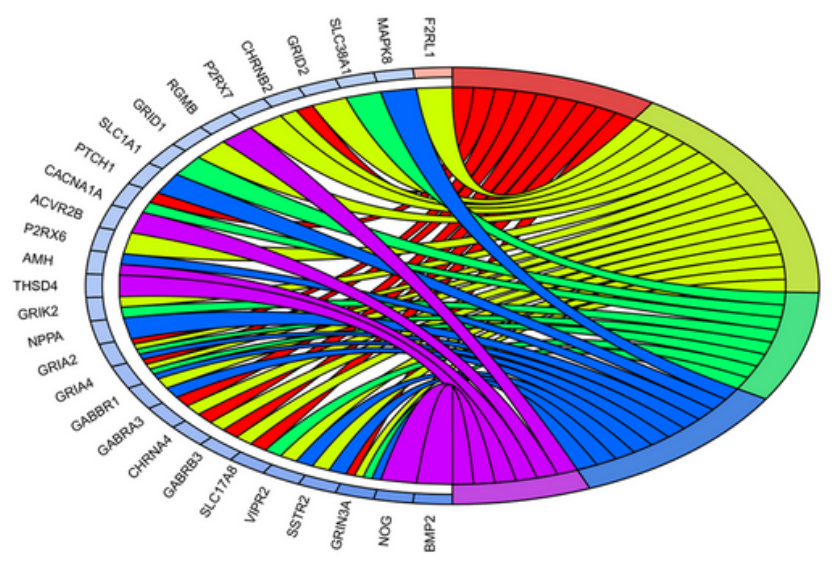

KEGG

Nicotine addiction $\square$ Neuroactive ligand receptor interaction

$\square$ Glutamatergic synapse $\square$ cAMP signaling pathway $\square$ TGF-beta signaling pathway

\section{Figure 4}

Functional enrichment analyses in the blue module. GO enrichment analysis in the (A) BP category, (B) CC category, (C) MF category; (D) KEGG pathway enrichment analysis. GO, gene ontology; BP, biological process; CC, cellular component; MF, molecular function; KEGG, Kyoto Encyclopedia of Genes and Genomes. 
A

B

LCAT + High LCAT + LOW LCAT

UST + High UST + Low UST
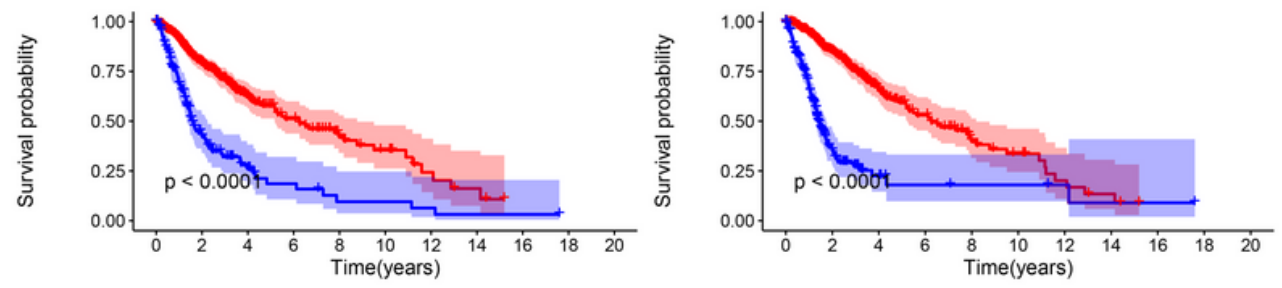

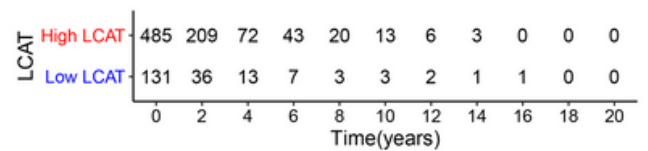

$\stackrel{5}{3}$ Low UST $\left\{\begin{array}{ccccccccccc}436 & 214 & 77 & 46 & 20 & 13 & 6 & 3 & 0 & 0 & 0 \\ 180 & 31 & 8 & 4 & 3 & 3 & 2 & 1 & 1 & 0 & 0 \\ \hline 0 & 2 & 4 & \dot{6} & \dot{8} & 10 & 12 & 14 & 16 & 18 & 20 \\ \text { Time(years) } & & & & \end{array}\right.$

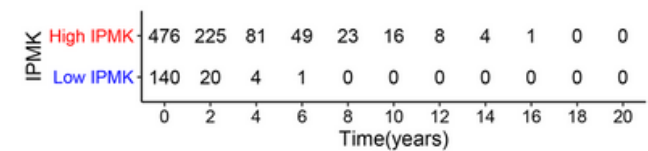

D

$\mathbf{E}$

NEU4 - High NEU4 - Low NEU4

GALNT13 - High GALNT13 - Low GALNT13
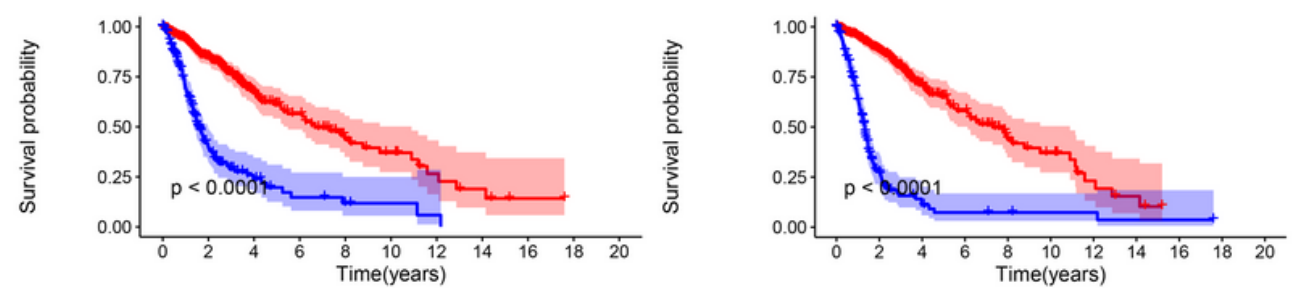

$\mathbf{F}$

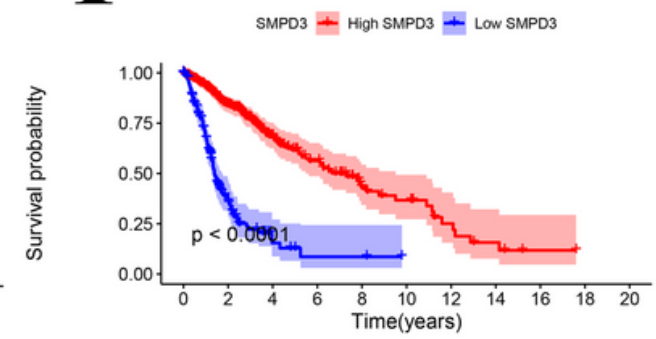

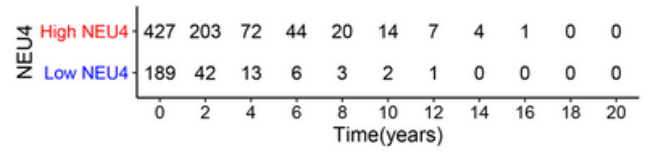

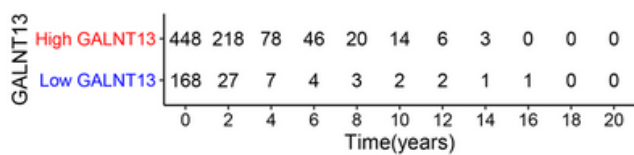

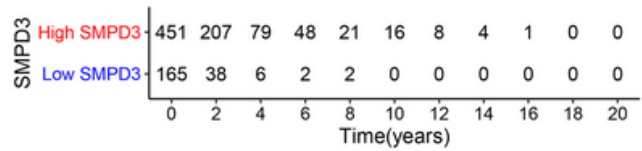

G

PDEGB + High PDEGB + LOW PDEGB

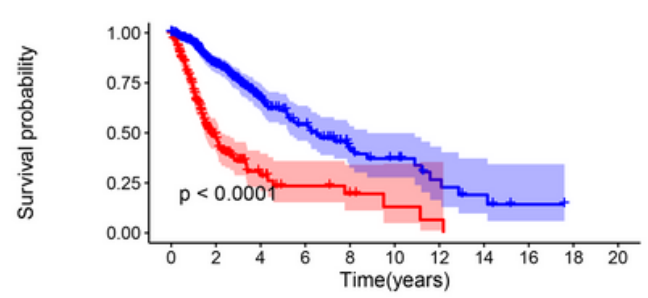

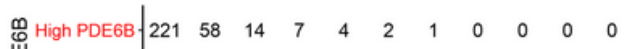

岩 Low PDE6B \begin{tabular}{cccccccccccc}
395 & 187 & 71 & 43 & 19 & 14 & 7 & 4 & 1 & 0 & 0 \\
\hline 0 & 2 & 4 & 6 & 8 & 10 & 12 & 14 & 16 & 18 & 20
\end{tabular}

\section{Figure 5}

Kaplan-Meier survival curves of seven core genes. Survival curve analysis of (A) LCAT expression (8.0); (B) UST expression (10.6); (C) IPMK expression (1.4); (D) NEU4 expression (5.1); (E) GALNT13 expression (6.3); (F) SMPD3 expression (1.3); (G) PDE6B expression (2.3) in relation to glioma patient OS. The values in brackets are their optimal cut-off values calculated by X-tile software. LCAT, Lecithin-Cholesterol Acyltransferase; UST, Uronyl 2-Sulfotransferase; IPMK, Inositol Polyphosphate Multikinase; NEU4, 
Neuraminidase 4; GALNT13, Polypeptide N-Acetylgalactosaminyltransferase 13; PDE6B, Phosphodiesterase 6B; SMPD3, Sphingomyelin Phosphodiesterase 3

A
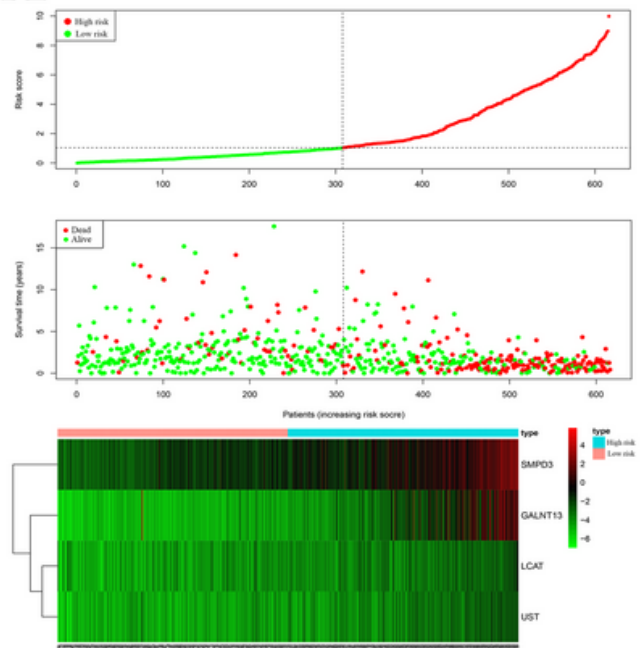

D

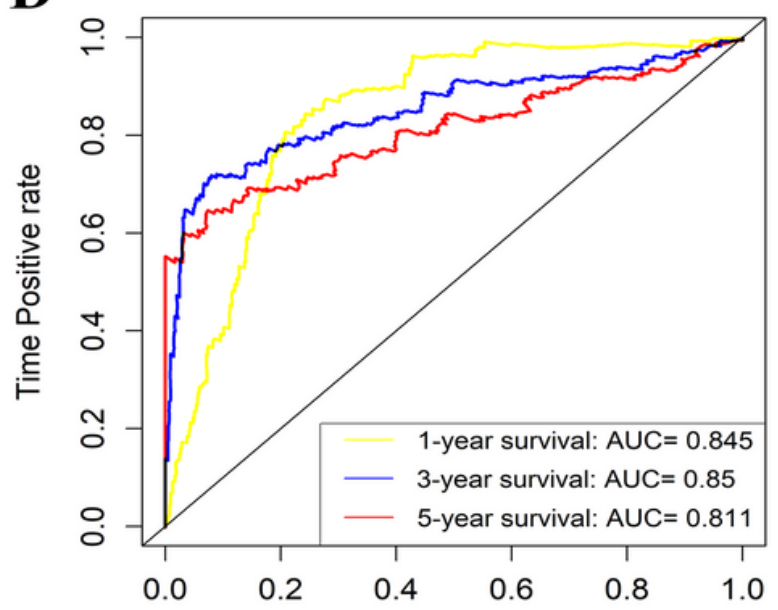

B
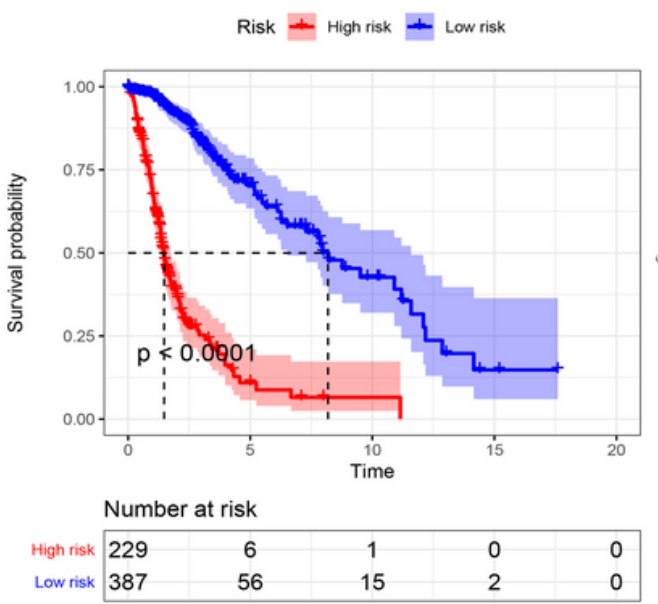

$\mathbf{E}$

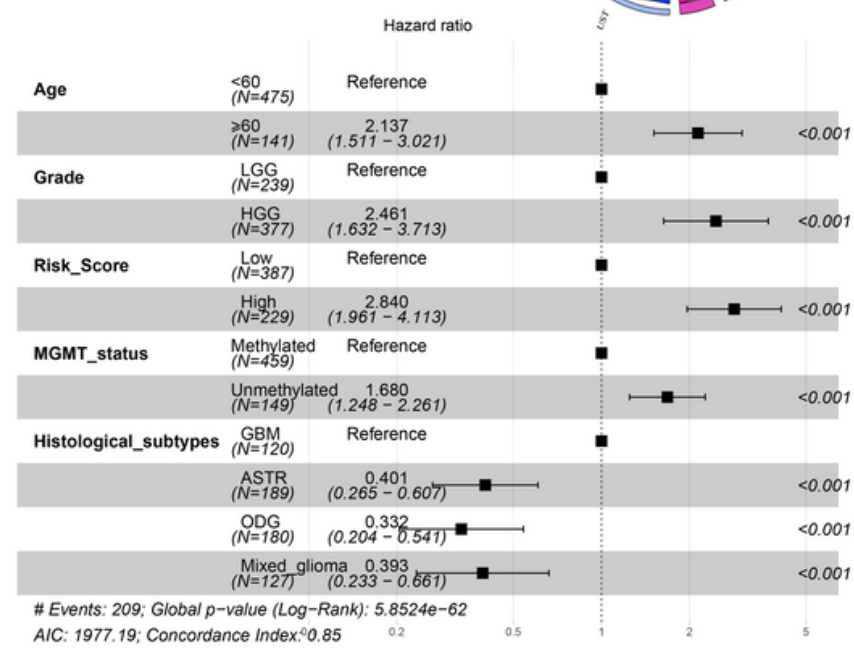

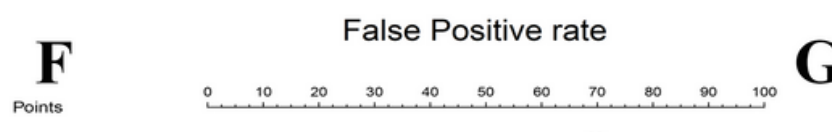
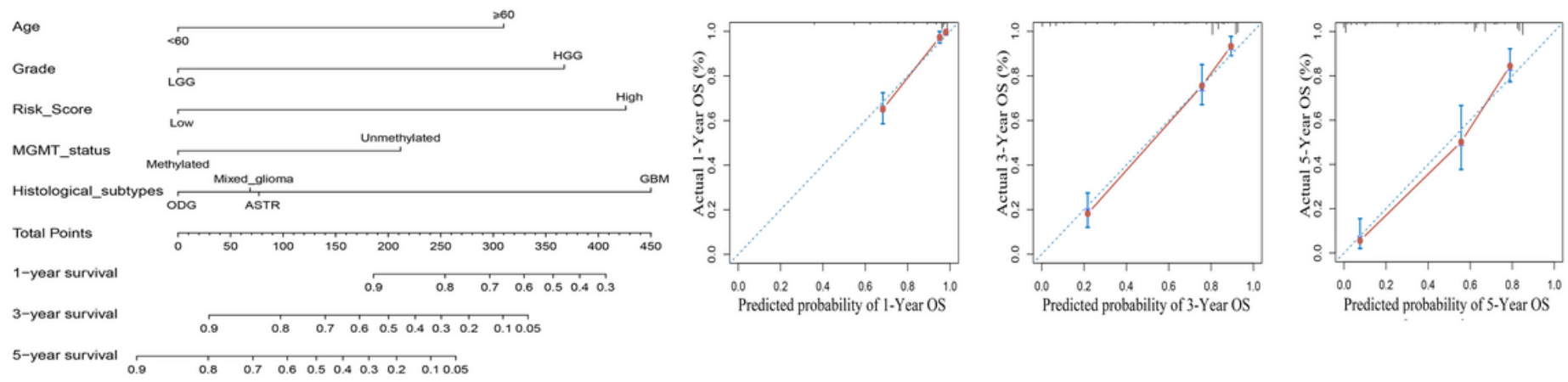

Figure 6

Construction and evaluation of a prognostic model in the TCGA training cohort. (A) The patient distribution, survival status and a heatmap of high/low risk score calculated by the prognostic model composed of four core genes; (B) Kaplan-Meier survival curve analysis of OS in patients with glioma on the basis of the four gene signature in the training cohort. The risk table shows the total number of 
patients at risk in the high/low risk group at the corresponding time; (C) GO Chord plot of four core gene enrichment analysis; (D) The time-dependent receiver operating characteristics (ROC) curves of the four gene signature for predicting 1-, 3-, and 5-year OS of glioma patients; (E) Multivariate cox proportional hazard regression analysis for the prognostic model and significant clinical traits; (F) Development of a nomogram to predict the 1-year OS, 3-year OS, and 5-year OS of glioma patients. (G) Calibration curves for evaluating the consistency between predicted probability and the actual probability for 1-year OS, 3year OS, and 5-year OS from nomogram. TCGA, The Cancer Genome Atlas; GO, gene ontology; BP, biological process; MF, molecular function; OS, overall survival; AIC, Akaike information criterion; AUC, area under the curve; LGG, low grade glioma; HGG, high grade glioma; ASTR, astrocytoma; ODG, oligodendroglioma; GBM, glioblastoma 

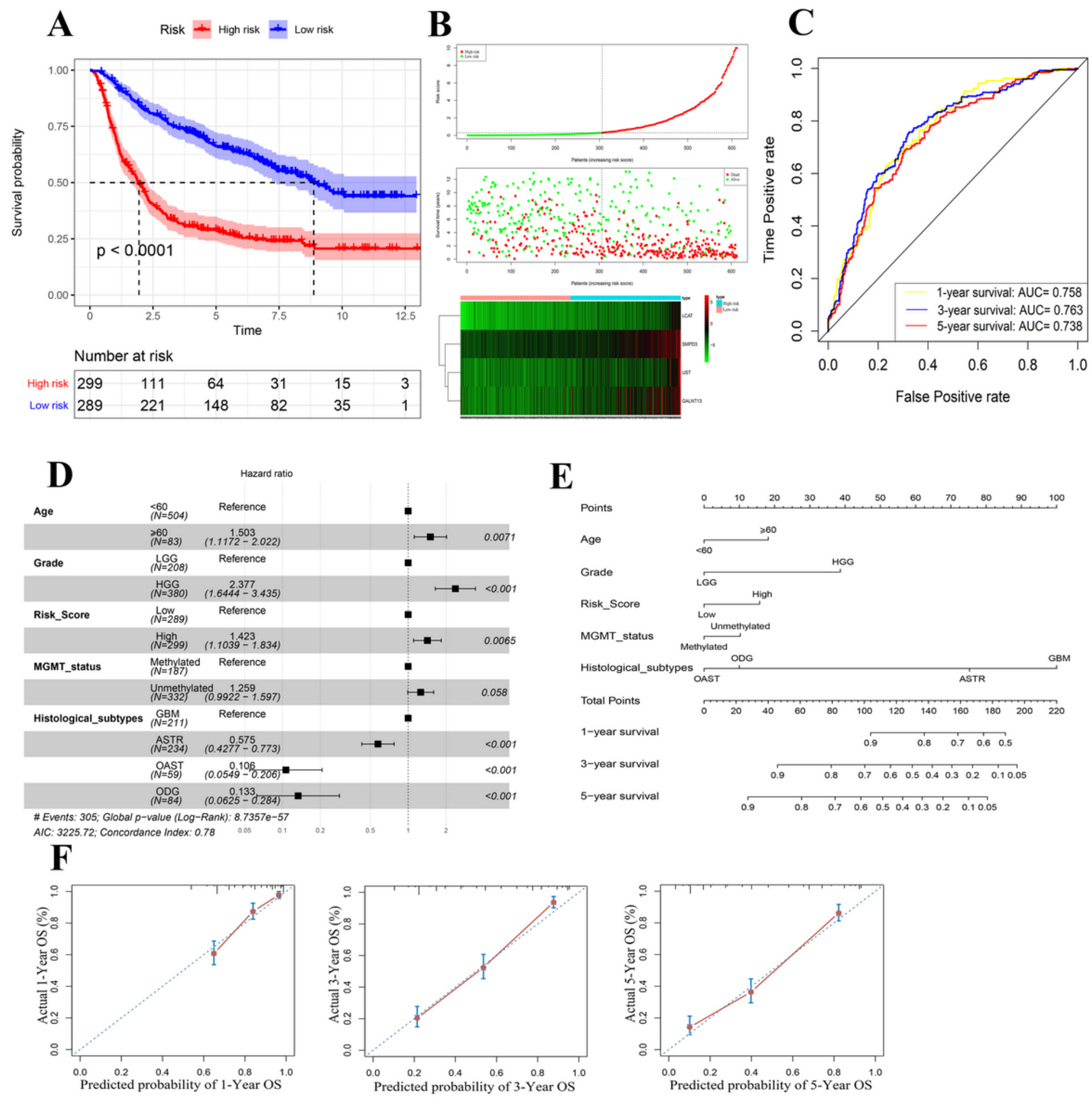

Figure 7

Validation of the four gene signature for the prediction of glioma survival in the CGGA validation cohort. (A) Survival curve showing the impact of risk score on glioma survival. (B) Risk plot, survival status of glioma patients, and heatmap of the four gene signature expression profile. (C) ROC curves of the four gene signature reveal its predictive performance for glioma survival at 1-year, 3-year and 5-year time points. (D)A forest plot demonstrating the prognostic model as an independent prognostic indicator of glioma patients. (E) A nomogram containing the risk score and essential clinical characteristics for 
prediction of the 1-year OS, 3-year OS, and 5-year OS of glioma patients. (F) Calibration plots assess the predictive capacity of the nomogram used to predict 1-year OS, 3-year OS, and 5-year OS of glioma. CGGA, the Chinese Glioma Genome Atlas; OS, overall survival; AIC, Akaike information criterion; ROC, receiver operating characteristics; AUC, area under the curve; LGG, low grade glioma; HGG, high grade glioma; ASTR, astrocytoma; ODG, oligodendroglioma; OAST, oligoastrocytoma; GBM, glioblastoma
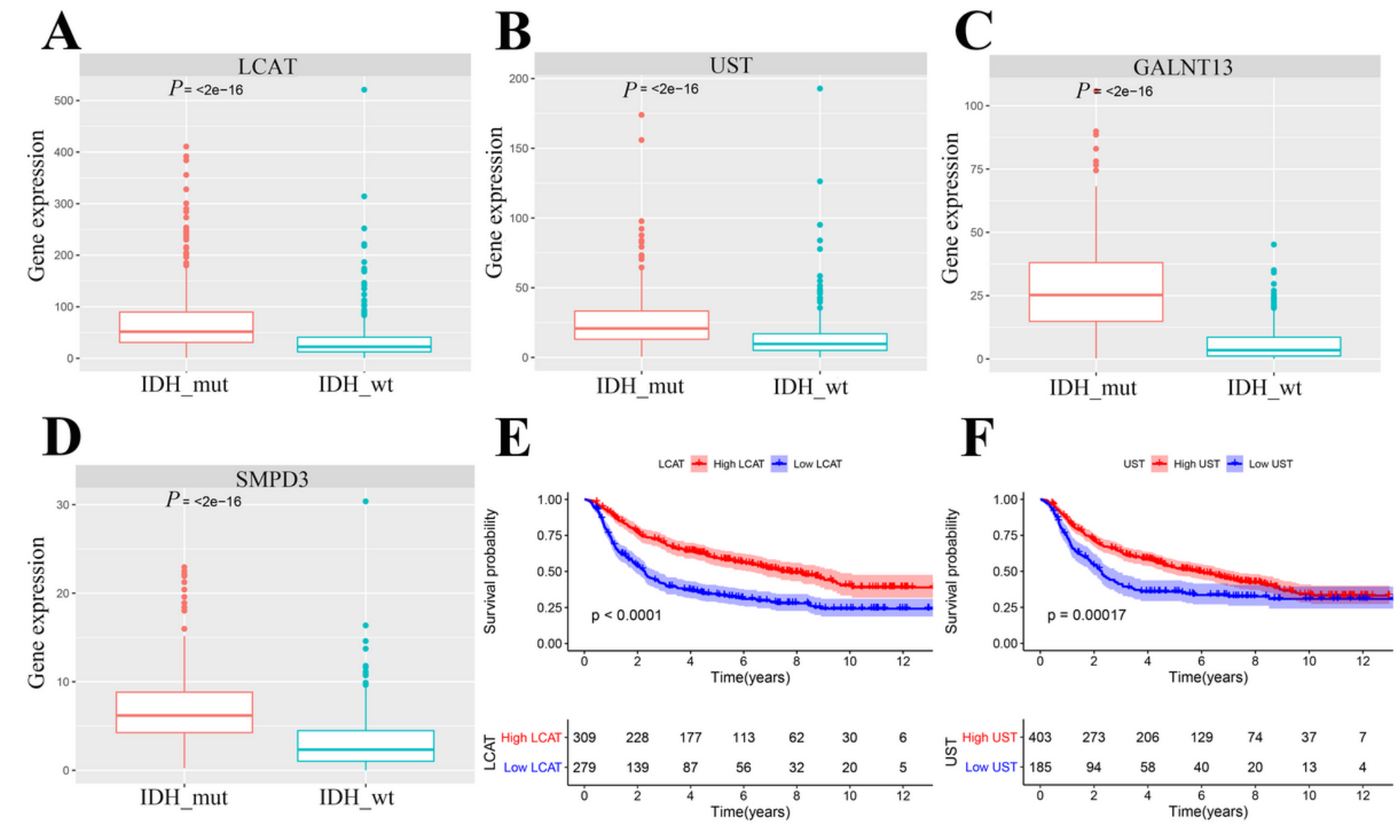

G

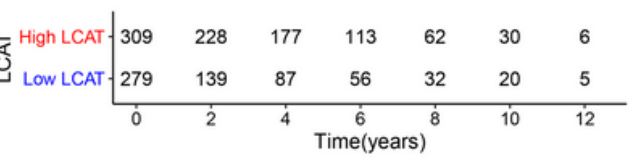

IDH_mut

IDH_wt

$\mathbf{H}$

GALNT13 + High GALNT13 - Low GALNT13
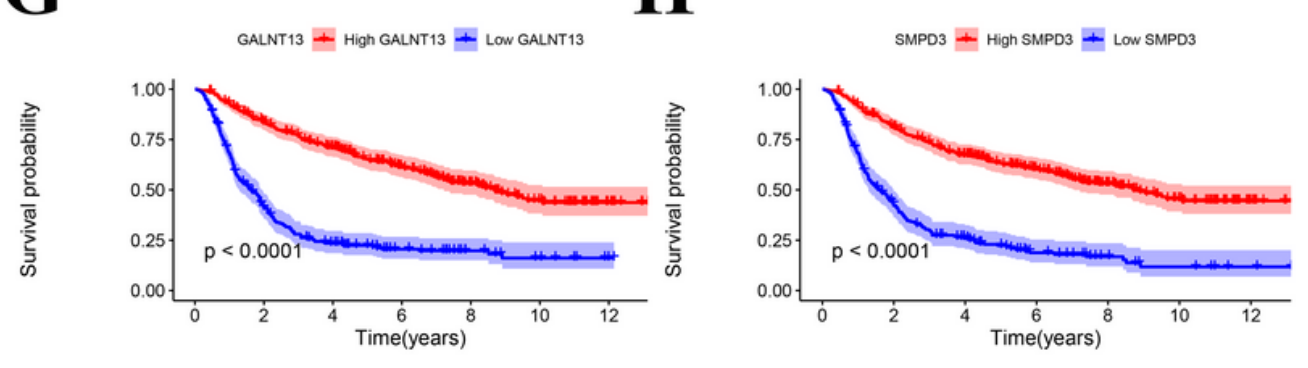

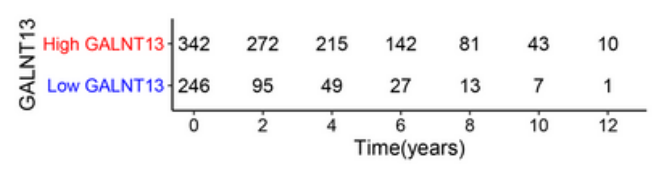

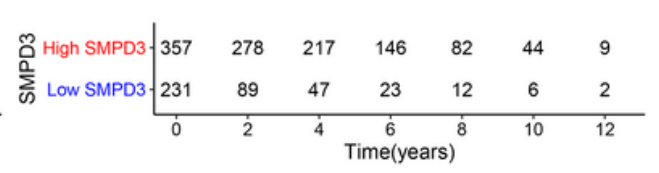

\section{Figure 8}


Assessment of the gene expression of four genes in different IDH status and their impact on glioma survival. Boxplot of (A) LCAT, (B) UST, (C) GALNT13 and (D) SMPD3 gene expression in IDH mutant and IDH wild-type glioma patients from CGGA cohort. Additionally, survival curves separately present the relationships between (E) LCAT, (F) UST, (G) GALNT13 and (H) SMPD3 gene expression and the OS of glioma patients derived from the CGGA database. IDH, isocitrate dehydrogenase; IDH_mut, IDH mutation; IDH_wt, IDH wild type; LCAT, Lecithin-Cholesterol Acyltransferase; UST, Uronyl 2-Sulfotransferase; GALNT13, Polypeptide N-Acetylgalactosaminyltransferase 13; SMPD3, Sphingomyelin Phosphodiesterase 3; OS, overall survival; CGGA, the Chinese Glioma Genome Atlas.

A
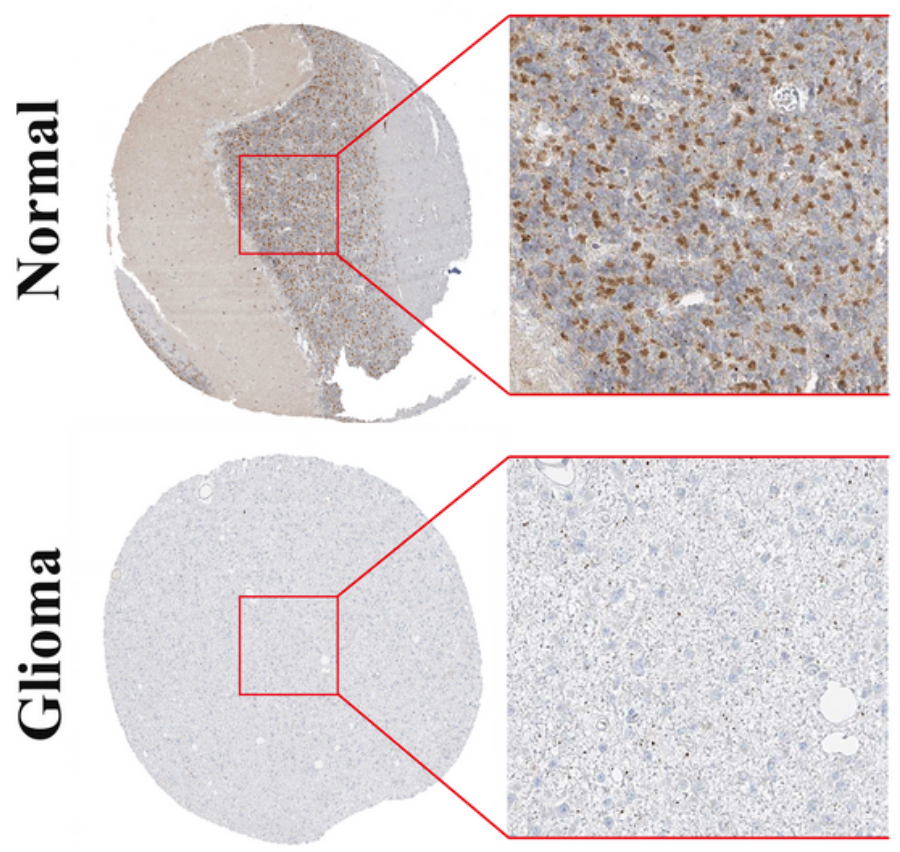

C
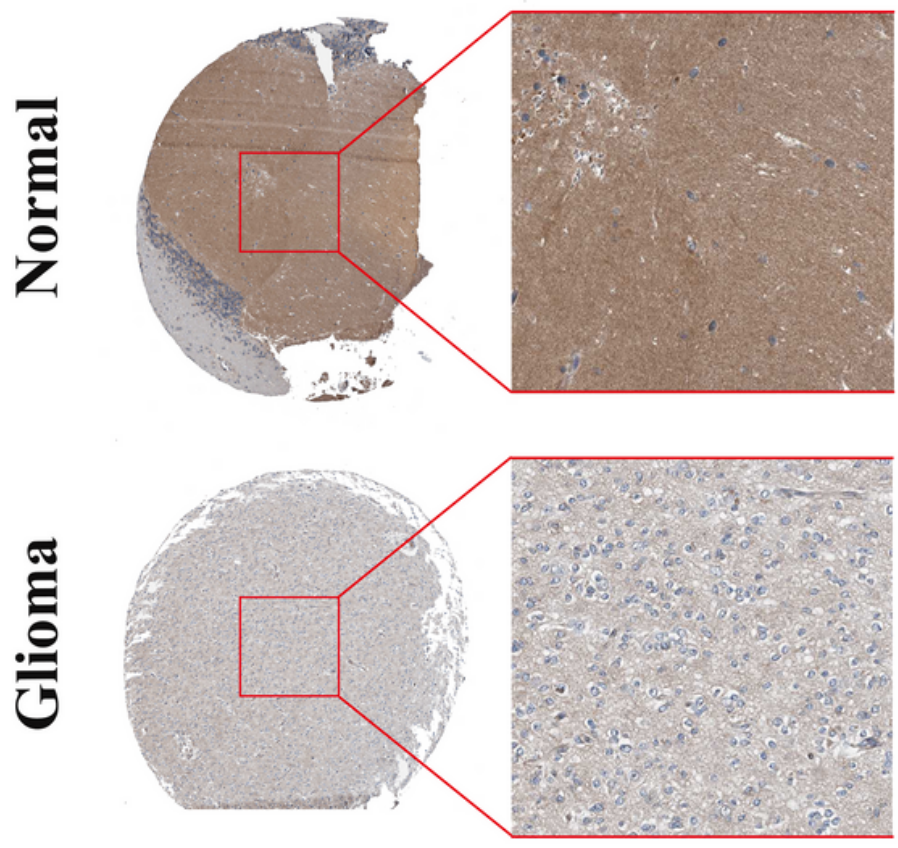

B
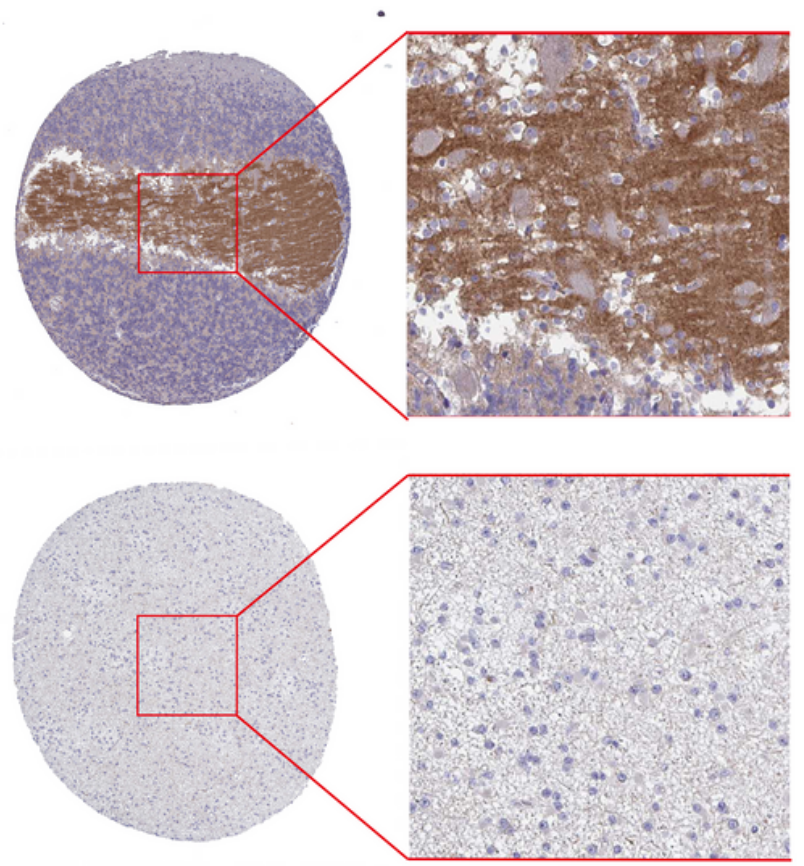

Figure 9 
The results of IHC staining for protein expression of core genes in the HPA database. The brown granule is representative of protein expression. IHC, immunochemistry; HPA, the human protein atlas; LGG, low grade glioma; HGG, high grade glioma; UST, Uronyl 2-Sulfotransferase; GALNT13, Polypeptide NAcetylgalactosaminyltransferase 13; SMPD3, Sphingomyelin Phosphodiesterase 3.

\section{Supplementary Files}

This is a list of supplementary files associated with this preprint. Click to download.

- SupplementaryTable1TheDEGs.xls

- OnlineSupplementaryFIGURE1.png

- OnlineSupplementaryFigure2.png 